Classification

Physics Abstracts

$47.25 \mathrm{Q}-61.30 \mathrm{G}$

\title{
Elementary and Composite Defects of Striped Patterns
}

\author{
A.C. Newell( $\left.{ }^{*}\right)$, T. Passot $\left({ }^{* *}\right)$, N. Ercolani and R Indik \\ Arızona Center for Mathematıcal Sciences, Department of Mathematıcs, Unversity of Arizona, \\ Tucson, AZ, 85721, USA
}

(Received 12 April 1995, revised and accepted 19 August 1995)

\begin{abstract}
Labyrinthic patterns are observed both in systems where the uniform states are metastable, as a result of a front instability, and in systems displaying a cellular instability, when the band of excited Fourier modes is wide enough to support resonant interactions between modes lying on different shells. We show that the phase formalism is a suitable description for low-density labyrunthic patterns with a relatively long range correlation and is capable of describing both its smooth and singular structures. The point defects of roll patterns, the concave and convex disclinations, and the line singularities or phase grain boundaries across which the wavevector makes an order one transition, are found to be singular and weak solutions of the Cross-Newell phase diffusion equation, which take account of their energetics as well as their topologies.
\end{abstract}

\section{Introduction}

Labyrinthic patterns are now widely observed in extended systems as different as Garnet films [1], chemical reactions [2,3], convection fluids at high Prandtl numbers [4], or optical beams [5]. Several mechanisms and models have been proposed to explain the formation of such patterns in the context of reaction-diffusion systems, all relying on the transverse instability of fronts $[6,7]$. They are often observed in bistable systems possessing two steady uniform states that are linearly stable. In this paper we show that labyrinthic patterns also arise in systems governed by an equation of the Swift-Hohenberg (S.H ) type when the stress parameter $R$ is large enough to have a wide annular band of excited wavevectors, but still at a value $(R<1)$ where the nonzero homogeneous states are linearly damped. In this range of stress parameter these systems usually display a lamellar structure, meaning a mosaic of patches of typical diameter $l$ of almost straight rolls joined together by phase grain boundaries that meet at point defects The first questions to be addressed concern the relation between the size of the patches and the stress parameter, as well as the time scale of the pattern evolution.

$\left(^{*}\right)$ Charre Condorcet, Ecole Normale Supérieure, Laboratoire de Physique, 24 rue Lhomond, Paris Cedex 05, France.

$\left(^{* *}\right)$ Permanent address. Observatorre de la Côte d'Azur, B P. 22906304 Nıce Cedex 4, France 
Lamellar structures with typical patch size $l$ (they will be called here labyrinthic when $l$ is of the order of a few roll wavelengths $\lambda$ ) become long lived above a certain value $R_{c}(l)$ of the applied stress. Below this value, they destabilize through an amplitude instability (e.g. reconnection of phase contours) on a short time scale. This property wll be illustrated on the simplest structures constituting these patterns, namely zig-zags, using a discrete mode analysis. The new feature is that multimode solutions become stabilized by resonant interactions between modes lying withın the annulus of excited wavevectors of width $\sqrt{R-R_{0}}$, where $R_{0}$ is the value of the stress parameter at which rolls (stripes) are first excited.

Above the critical value $R_{c}(l)$, lamellar structures undergo mainly phase adjustments which take place on time scale long with respect to the amplitude time scale (for $R-R_{0} \gg \epsilon^{2}=(\lambda / l)^{2}$, the evolution of the phase, which because of translational invariance is still a soft or Goldstone mode of the system, is slow and of order $\epsilon^{-2}$, whereas the fluctuations in amplitude about its slaved value occur on the $\left(R-R_{0}\right)^{-1}$ time scale) It is thus natural to investigate whether the phase diffusion formalism provides an appropriate description. The main question naturally concerns the presence of defects, leading to singularities of the wavevector and multivaluedness of the phase. In this paper we show that even these singular structures are well captured by the (single roll) Cross-Newell phase diffusion equation,

$$
\tau(k) \theta_{t}+\nabla \cdot \mathrm{k} B(k)+\epsilon^{2} \mathcal{R}=0,
$$

which governs the evolution of the phase of some microscopic field $w=F(\Theta=(1 / \epsilon) \theta)$, with $\epsilon=\lambda / l$ and $F(\Theta)$ a $2 \pi \epsilon^{-1}$ periodic function of $\Theta$. The validity of this large scale equation extends to values of $\epsilon^{-1}$ of a few units, namely for low density labyrinthic patterns. It is a universal description in the sense that the microscopic details enter only in the exact form of the phase diffusion coefficients, but not in the form of the equation itself This equation is simply a solvability condition that arises directly from the property of local translational invarıance which makes the phase a neutral mode of the system (for details see [8.9]). In (1), $k$ is $|\nabla \theta|$, the functions $\tau(k, R), B(k, R)$ are calculable from the underlying microscopic system. Typically in $\left(k_{1}, k_{\mathrm{r}}\right)$, the range of wavenumbers where a finite amplitude roll solution exists, $k B$ has the shape of a cubic curve with zeros at $k_{1}, k_{\mathrm{r}}$, an intermediate zero at $k_{\mathrm{B}}$, the wavenumber selected by curved rolls [8], a maximum at $k_{\mathrm{El}}<k_{\mathrm{B}}$ (left Eckhaus boundary) and a minimum at $k_{\mathrm{E}}>k_{\mathrm{B}}$ (right Eckhaus boundary) (see Fig. 1). The stability properties of roll solutions can be understood in terms of the type of the spatial operator $\nabla \cdot \mathrm{k} B$ It is a second order, quasilinear operator in spatial derivatives acting on $\theta$. It is elliptic negative (positive) for $k$ in $\left(k_{\mathrm{B}}, k_{\mathrm{E}}\right),\left(k_{1}, k_{\mathrm{El}}\right)$, hyperbolic in $\left(k_{\mathrm{El}}, k_{\mathrm{B}}\right)$ and $\left(k_{\mathrm{E}}, k_{\mathrm{r}}\right)$ Thus (1), with $\epsilon=0$, 1s ill-posed as an initial value problem outside of the Busse balloon $\left(k_{\mathrm{B}}, k_{\mathrm{E}}\right)$ and appropriate regularization terms must be added [9]. For $k<k_{\mathrm{B}}$, the zig-zag instability increases the roll wavenumber by bending. It is supercritical and it is saturated by the addition of $\mathcal{R}=e \frac{\left|B^{\prime}\left(k_{\mathrm{B}}\right)\right|}{4 k_{\mathrm{B}}} \nabla^{2} \nabla \mathbf{k}$, where $e=1$ for $k \simeq k_{\mathrm{B}}$. For $k>k_{\mathrm{E}}$, the Eckhaus instability decreases the wavenumber by the nucleation of a pair of dislocations and removal of a roll pair This instability is subcritical, does not saturate in a nearby state, and requires the reintroduction of the amplitude $A(k)$, almost everywhere slaved to $k$, as an active order parameter [9]. Amplitude regularization is also required in the cores of point defects $[10,11]$

In Section 2, we report on numerical simulations of the S.H. equation at different values of the stress parameter $R$ and discuss a possible mechanism for the stabilization of spatially random lamellar structures, paying particular attention to undulating rolls and zig-zags, which constitute the basic smooth elements of lamellar structures. Section 3 is devoted to an analysis of the point and line defects of these lamellar patterns. The building blocks of all point defects, the concave and convex disclinations, composites of which form the saddle, the tar- 


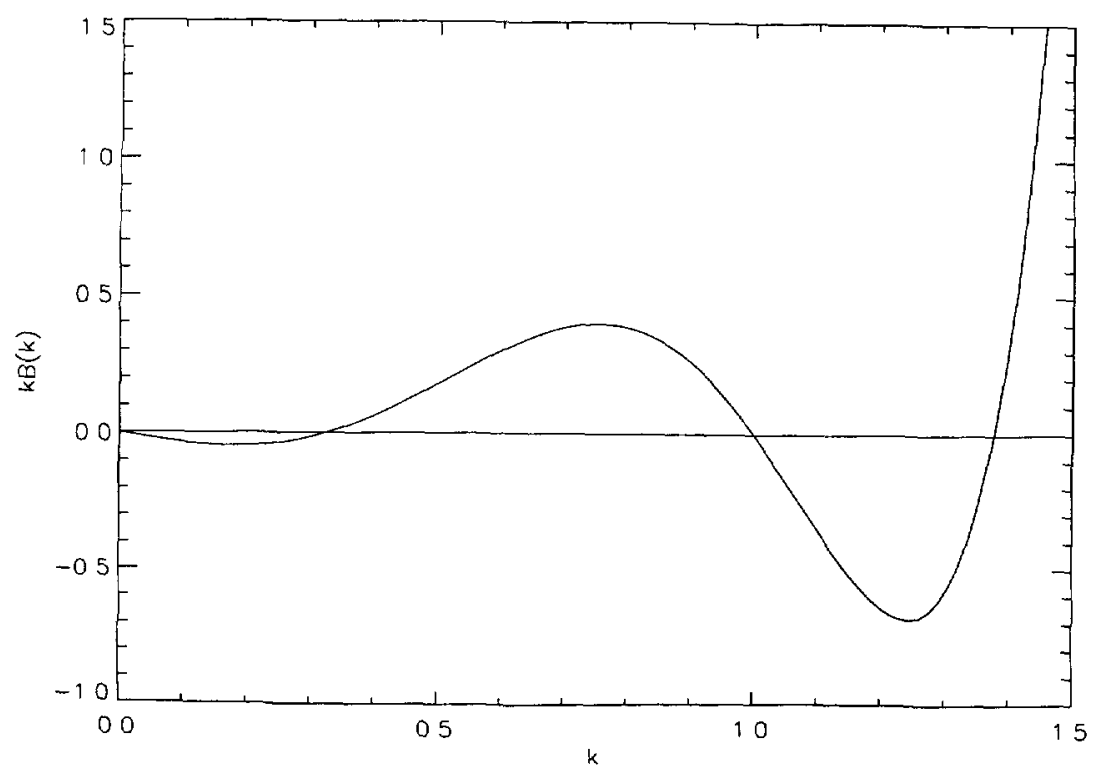

Fig 1. - Plot of $k B(k)$ for $B(k)=2\left(1-k^{2}\right)\left(R-\left(1-k^{2}\right)^{2}\right)$, with $R=0.8$ This form of $B(k)$ is obtained with the complex S H. equation We have $k_{1}=\sqrt{1-\sqrt{R}} \approx 032, k_{\mathrm{El}} \approx 0.75, k_{\mathrm{B}}=1$, $k_{\mathrm{E}} \approx 124$ and $k_{\mathrm{r}}=\sqrt{1+\sqrt{R}} \approx 1.38$ Although drawn for all $k$, it is only meaningful for $k_{l}<k<k_{r}$

get, the vortex, type I and II handles, the dislocation and the bridge, are new singular and weak solutions of the Cross-Newell (C.N.) phase diffusion equation (1.e. when $\mathcal{R} \rightarrow 0$ ). These solutions capture the observed topology of defects. In addition, they accurately describe the energetic properties in the limit of infinite aspect ratios. Emerging from each concave disclination are three line defects, each phase grain boundary, across which the phase is continuous and its gradient discontinuous. These are also weak solutions of the C.N. equation. We also present a new class of solutions of the regularized phase diffusion equation which approximate the exact solutions in most cases of interest, and which are a generalization of the linearization already proposed in [12] in the particular context of slight deviations to straight rolls Finally, a summary is presented in Section 4 .

\section{Numerical Simulations and Models of Zig-Zags}

2.1. Numerical Results - We solve the Swift-Hohenberg equation, $w_{t}+\left(\nabla^{2}+1\right)^{2} w-$ $R w+w^{3}=0$, in a periodic domain of $128 \times 128$ grid points, using a standard spectral method. The size of the domain is exactly 20 roll wavelengths at the critical wavenumber $k=1$. The horizontal diffusion time thus corresponds to about 400 time units. Starting with random noise, we let the system evolve for several (about 5 ) horizontal diffusion times. Several values of the stress parameter $R$ will be used Special values of $R$ are $R=0$ above which rolls at $k=1$ get first excited, $R=1$ above which the uniform states $w= \pm \sqrt{R-1}$ are linearly excited and $R=1.5$ above which these flat states become linearly stable. In Figure 2, we show different patterns for $\vec{R}=0.4,0.6,0.97$ and 1.5 at times $t=2000$. The bright (dark) areas correspond to large positive (negative) values of $w$. Whereas for $R=0.97$ and $R=1.5$, 

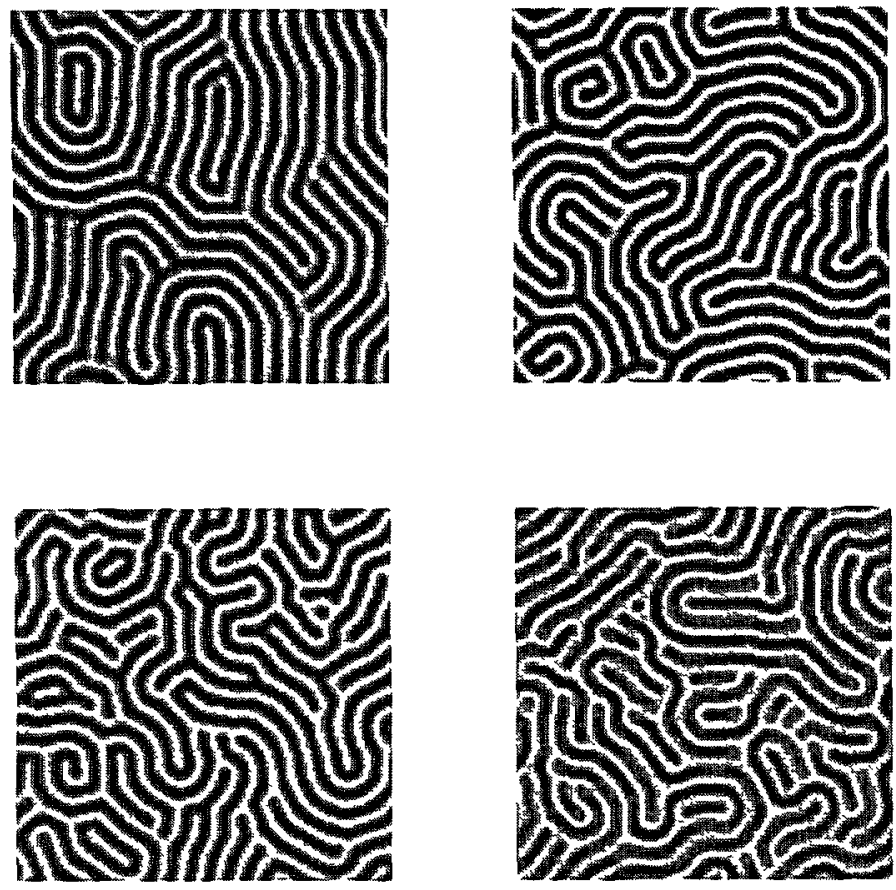

Fig. 2. - Quas1-stationary patterns obtained with the S.H. equation for $R=0.4$ (a: top left), $R=06$ (b top right), $R=0.97$ (c bottom let) and 1.5 (d: bottom right) at $t=2000$.

the only visible changes observed between $t=1000$ and $t=2000$ are a slight modification of the angles between adjacent rolls, for $R=0.4$ and $R=0.6$, we still observe slight topological changes.

Several remarks can be made:

1) The pattern correlation length, which is inversely proportional to the width of the annulus of excited Fourier modes, is smaller when the stress parameter is increased, consistent with other results we will predict This has also been observed by Morris et al. [13] in experiments and by Decker et al. [4] in simulations of the Oberbeck-Boussinesq equations at moderate Prandtl number For low values of $R$ (typically $R<0.1$ ), defects consist mostly of dislocations and grain boundaries, and the pattern evolves until it consists of straight parallel rolls. For $R=0.4$ and $R=0.6$ the quasi-stationary patterns contain a few disclinations. In a smaller domain and with an inverse aspect ratio of 10 instead of 20 , we observed at $R=04$, immobile disclinations for as long as we continued the run, i.e. up to 100 horizontal diffusion times $(t=10000)$.

2) For larger values of $R$ ( $R=0.97$ and 1.5). the correlation length is of the order of a few roll wavelengths, and there are many isolated free disclinations. The pattern can then be called labyrinthic, but we stress that (even when $R=1.5$ ) it has been formed as a result of a cellular instability at $k=1$. In the case $R=1.5$, large scale modulations of the amplitude can be detected (likewise in Fig. 3f of [2]). The symmetry $w \rightarrow-w$ is broken, as revealed by the fact that all convex disclinations are of the same type: the roll emanating from the defect core is such that $w<0$ (a similar observation is also made in [1]). These facts indicate that a significant amount of energy has built up near the $k=0$ mode, which has a role to play but not a dominant role. Since the system is variational (it has been reported in [14] that nonvariational 


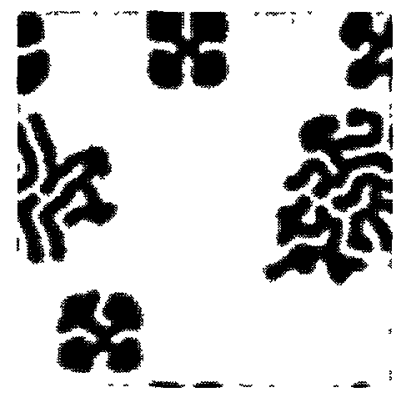

Fig 3. - Transient pattern of the S H. equation at $R=25$.

effects are not crucial for the formation of labyrinthic patterns but note nevertheless, that nonvariational effects can also destroy labyrinthic patterns [7]), it has probably reached a local minimum of the free energy, although it is very hard to confirm this numerically. The slow evolution of the pattern seems to be the result of phase generation or absorption at the cores of convex disclinations, whose amplitudes are observed to vary periodically in time with a period of approximately one horizontal diffusion time. At the present time, we cannot offer any reasonable explanation for this time dependence, although we suspect that it is the result of a frustration in wavenumber (see also [15]). Let us mention here that, although concave and convex disclinations are topological counterparts, they are not energetically equivalent. Concave disclinations are locked in place by shocks and the amplitude does not come into play. They belong to regions in $k$ space $\left(k<k_{\mathrm{B}}\right)$ where phase regularization is sufficient. Convex disclinations have $k>k_{\mathrm{B}}$ at their core and the amplitude is more likely to be a free mode in the center.

3 ) In Figure 3 we show a transient at $R=25$. Now the zero mode is all important. The initial bubbles of $w= \pm \sqrt{R-1}$ that emerge from the noise undergo a front instability [16] when their diameter is large enough and rolls grow from their boundary, ultimately invading the whole domain and forming a maze. The mode $k=0$, corresponding to the stable uniform states $w= \pm \sqrt{R-1}$, definitely plays a role in the process leading to this labyrinthic pattern, unlike for the cases $R<1$, where only a shell of modes around $k=1$ is excited. In this parameter range, the behavior of the S.H. equation is very similar to that observed in other bistable systems $[6,7]$.

Such disordered patterns can also be obtained as a result of a zig-zag instability on straight rolls (see [1]). We performed a numerical experiment starting with 15 rolls whose phase contains a small random noise and whose wavenumber corresponds to $k=0.7$, far into the zig-zag unstable band. Figure 4 displays the outcome of the instability (also almost stationary), for $R=0.97$ and 1.5 . For $R=0.4$ (not shown) the final state consists mainly of straight rolls. For $R<1$, the resulting pattern is very similar to those obtaned with random initial conditions (see Fig. 4a). However, for $R=1.5$ rolls act as individual fronts which avoid reconnecting, leading to this very different looking structure of "hairy rolls" shown in Figure 4b.

We now argue that the persistence of labyrinthic patterns far from onset is due to resonant interactions between modes that he both on the circle $|k|=1$ and inside this circle. When the stress parameter is too small, the growth rates of the modes lying inside the circle do not overcome the nonlinear damping due to coupling with other faster growing modes and thus the competition between modes on the circle ultimately leads to a structure dominated by a single roll. For larger values of $R$, modes lying inside the circle can be part of a four wave interaction that stabilizes a configuration involving several modes on the circle and several modes inside 

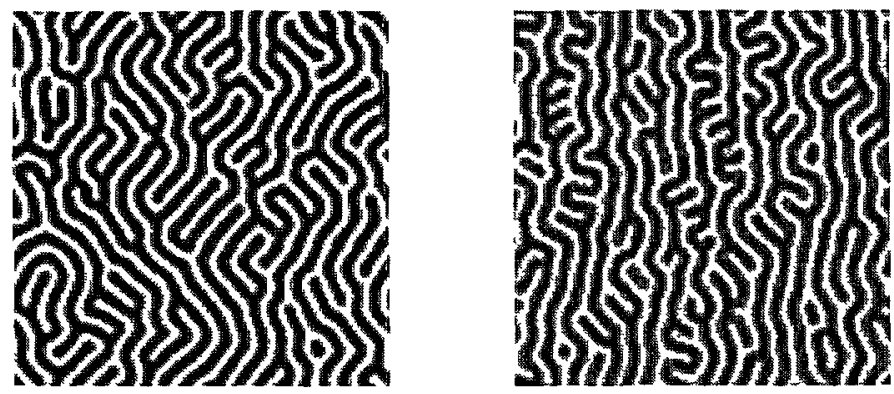

Fig 4. - States obtained from a zig-zag instability $\left(k_{0}=0.7\right)$ of straight rolls at $R=0.97$ (a. left) and $R=1.5$ (b right)
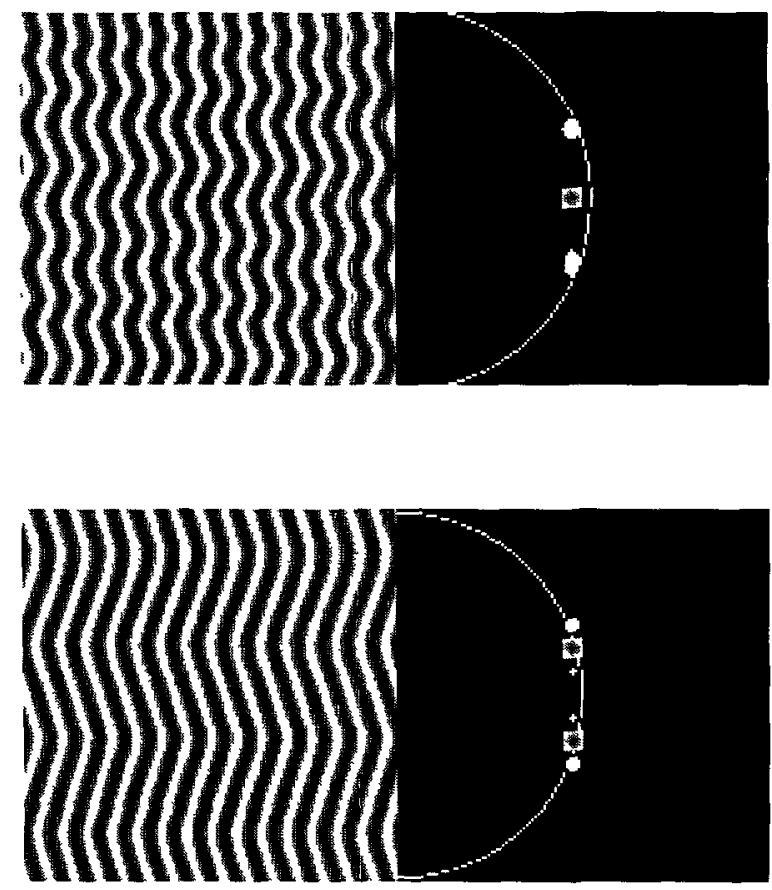

Fig. 5 - States obtained from a zıg-zag instability $\left(k_{0}=09\right.$ ) of stralght rolls at $R=0.97$ (a: top) (and for $k_{0}=0.95, R=0.2$ (b: bottom)) The scale is such that 15 rolls at $k=1$ fit exactly in the domain The Fourier spectrum (for $k_{x}>0$ ) as well as the critical circle are displayed on the right.

it. Since lamellar patterns involve spatial locality and are thus difficult to model in Fourier space, we illustrate this phenomenon with several simple solutions displaying undulating rolls, zig-zags and arrays of disclinations.

2.2. Projection on Modal Equations. - Figure 5a shows a typical snapshot of the time evolution of a set of zig-zag unstable rolls at $R=0.97$, with an initial wavenumber $k_{0}=0.9$ The initial condition is $w=\cos \left(k_{0} x+r(x, y)\right)$, where $r$ is a small random perturbation. As seen in the Fourier spectrum displayed besides the pattern, the solution in Figure 5a is almost 
entirely made up of three modes, $A_{1}, A_{2}$ and $C$ (and their complex conjugates). The $C$ mode corresponds to the initial condition and the $A$ modes lie on the critical circle at a location where $\mathbf{k}_{C}$ bisects the angle between $\mathbf{k}_{A_{1}}$ and $\mathbf{k}_{A_{2}}$. In Figure $5 \mathrm{~b}$, we display a set of zig-zags obtained at $R=0.2$ with the same initial conditions as for Figure $5 \mathrm{a}$. In that case the system consists mostly of four modes, $A_{1}, A_{2}$, at the intersection of the circle $k=1$ and the axis $k_{x}=k_{0}$ (here $k_{0}=0.95$ ), and $B_{1}, B_{2}$, symmetric with respect to the axis $k_{y}=0$, still on the axis $k_{x}=k_{0}$ but inside the critical circle. (Note also the presence of two other inner modes on this line, whose amplitude is much smaller).

Undulating rolls and zig-zags of various angles and wavelengths can thus be well represented by the five modes $A_{1}, A_{2}, B_{1}, B_{2}$ and $C$, with symmetry about the axis $k_{y}=0$. A dynamical system for the amplitudes of these modes is easily derived from the free energy of the reduced system, obtained from that of the $\mathrm{S} \mathrm{H}$. equation:

$$
\mathcal{F}=\int \mathrm{d} x \mathrm{~d} y\left\{-\frac{1}{2} R w^{2}+\frac{1}{4} w^{4}+\frac{1}{2}\left(\left(1+\Gamma^{2}\right) w\right)^{2}\right\},
$$

after inserting $w=A_{1} e^{\imath \mathbf{k}_{A_{1}} \mathbf{x}}+\cdots+$ c.c.. In the case of an infinite domain (and pure Fourier modes), only "pure resonant interactions" remain and the model equations are simply e.g. $\mathrm{d} A_{\imath} / \mathrm{d} t=-\delta F / \delta \bar{A}_{\imath}$ where

$$
\begin{aligned}
F= & -\left(\sigma_{a}\left(\left|A_{1}\right|^{2}+\left|A_{2}\right|^{2}\right)+\sigma_{b}\left(\left|B_{1}\right|^{2}+\left|B_{2}\right|^{2}\right)+\sigma_{c}|C|^{2}\right. \\
& -3\left(\left|A_{1}\right|^{2}+\left|A_{2}\right|^{2}+\left|B_{1}\right|^{2}+\left|B_{2}\right|^{2}+|C|^{2}\right)^{2} \\
& +\frac{3}{2}\left(\left|A_{1}\right|^{4}+\left|A_{2}\right|^{4}+\left|B_{1}\right|^{4}+\left|B_{2}\right|^{4}+|C|^{4}\right) \\
& \left.-6\left(\bar{A}_{1} \bar{A}_{2} B_{1} B_{2}+\text { c.c. }\right)-3\left(\bar{A}_{1} \bar{A}_{2} C^{2}+\text { c.c. }\right)-3\left(\bar{B}_{1} \bar{B}_{2} C^{2}+\text { c.c. }\right)\right) .
\end{aligned}
$$

We write $\sigma_{e}=R-\left(1-k_{e}^{2}\right)^{2}$, with $k_{e}(e=a, b, c)$ denoting the wavenumbers of the modes $A_{\imath}, B_{\imath}$ and $C$ respectively (note that $k_{\mathrm{c}} \equiv k_{0}$ ). Stationary solutions of the dynamical system derived from (3) where the five modes are simultaneously non-zero, are unstable. Indeed, the system for the phases $\Phi_{2}=2\left(\phi_{a}-\phi_{b}\right), \Phi_{3}=2\left(\phi_{b}-\phi_{c}\right),\left(\Phi_{1}=\Phi_{2}+\Phi_{3}\right)$, reads

$$
\begin{aligned}
& \frac{\mathrm{d} \Phi_{2}}{\mathrm{~d} t}=\left(12 b^{2}+12 a^{2}\right) \sin \Phi_{2}-6 c^{2} \sin \Phi_{3}+6 c^{2} \sin \Phi_{1} \\
& \frac{\mathrm{d} \Phi_{3}}{\mathrm{~d} t}=\left(6 c^{2}+12 b^{2}\right) \sin \Phi_{3}-12 a^{2} \sin \Phi_{2}+12 a^{2} \sin \Phi_{1}
\end{aligned}
$$

where $a, b, c$ and $\phi_{e}(e=a, b, c)$ are respectively the moduli and the phases of the $A_{\imath}, B_{\imath}$ and $C$ modes. The only stationary solutions of this system are such that $\sin \Phi_{1}=\sin \Phi_{2}=\sin \Phi_{3}=0$ Among the four possibilities: $\Phi_{1}=\Phi_{2}=\Phi_{3}=0 ; \Phi_{1}=\Phi_{2}=\pi, \Phi_{3}=0 ; \Phi_{1}=\Phi_{3}=\pi, \Phi_{2}=0$ and $\Phi_{2}=\Phi_{3}=\pi, \Phi_{1}=0$, the first one is phase unstable, as can be seen immediately from (4)-(5) and the other three are amplitude unstable, as can be seen by inspecting the Hessian of $F$ at the corresponding solution $(a, b, c)$. As a consequence, the only possible solutions of this system, containing more than one non-zero mode, are those where either $C=0$ or $B_{1}=B_{2}=0$ (or $A_{1}=A_{2}=0$, but this is clearly unstable).

To study their relative free energy and local stablity properties, one must be able to deal with the limiting case where $B_{1}, B_{2}$ are very close to each other. As the two $B$ modes merge the free energy (3) undergoes a jump. Continuity is recovered if one considers "quasi-resonances", 1.e. finite domain or wave packet effects. In finite but large geometries, resonances do not have to be perfectly sharp and indeed a resonance can still play an important role even though its effect is weakened by a factor containing the detuning parameter. Let us give a simple expression for this detuning parameter $\gamma_{\imath}$, (the index $\imath$ referring to two wave interactions when $\imath=1$ or four 
wave interaction when $\imath=2$ ) in the case where the integration occurring in (2) is over a finite, but large, domain $-L \leq x \leq L,-L \leq y \leq L,(L \gg 1)$, and in the case where the modes are Gaussian wavepackets, instead of pure Fourier modes. A term of the form $\left(1 / 4 L^{2}\right) \int \mathrm{d} x \mathrm{~d} y w^{2} / 2$ in the free energy, leads, in addition to pure resonant terms $\left|B_{1}\right|^{2}$ and $\left|B_{2}\right|^{2}$, to cross terms of the form $\left(1 / 4 L^{2}\right) \int \mathrm{d} x \mathrm{~d} y \vec{B}_{1} B_{2} \mathrm{e}^{2\left(\mathbf{k}_{B_{1}}-\mathbf{k}_{B_{2}}\right) \mathbf{x}}=\bar{B}_{1} B_{2}(\sin K L) / K L=\gamma_{1} \bar{B}_{1} B_{2}$ (where we have set $\mathbf{k}_{B_{1}}-\mathbf{k}_{B_{2}}=K \mathbf{e}_{y}$ ) which contribute when $K$ is small. All the other terms involving $B_{1}^{2}$, $\ldots$, are negligible. When considering Gaussian wave packets instead of pure Fourier modes, writing each mode in the form $\sqrt{\alpha / \pi} B_{\imath} \mathrm{e}^{\imath \mathbf{k}_{B_{\imath}}} \mathbf{x}-(\alpha / 2) \mathbf{x}^{2}$, the term $\int_{-\infty}^{\infty} \mathrm{d} x \mathrm{~d} y w^{2} / 2$ gives rise (in an infinite domain), to an additional contribution of the form $\mathrm{e}^{-K^{-2} /(4 \alpha)} \bar{B}_{1} B_{2}$. This defines $\gamma_{1}=\mathrm{e}^{-K^{2} /(4 \alpha)}$ and, after renormalization of the amplitudes, the term $\gamma_{2}$ appearing in front of the quartic terms $\bar{B}_{1} \vec{B}_{2} B_{1} B_{2}$, will read $\gamma_{2}=\gamma_{1}^{2}$.

The free energy of the five mode system thus involves a lot of additional terms, and is quite complicated to write down. But, as we have just seen, we need only to consider the case of the four modes $A_{1}, A_{2}, B_{1}, B_{2}$ for which the free energy is

$$
\begin{aligned}
F= & -\left(\sigma_{a}\left(\left|A_{1}\right|^{2}+\left|A_{2}\right|^{2}\right)+\sigma_{b}\left(\left|B_{1}\right|^{2}+\left|B_{2}\right|^{2}+\gamma_{1}\left(\bar{B}_{1} B_{2}+B_{1} \bar{B}_{2}\right)\right)\right. \\
& -3\left(\left|A_{1}\right|^{2}+\left|A_{2}\right|^{2}+\left|B_{1}\right|^{2}+\left|B_{2}\right|^{2}\right)^{2}+\frac{3}{2}\left(\left|A_{1}\right|^{4}+\left|A_{2}\right|^{4}+\left|B_{1}\right|^{4}+\left|B_{2}\right|^{4}\right) \\
& -6\left(\bar{A}_{1} \bar{A}_{2} B_{1} B_{2}+\text { c.c. }\right)-3 \gamma_{1}\left(\bar{A}_{1} \bar{A}_{2}\left(B_{1}^{2}+B_{2}^{2}\right)+\text { c.c. }\right) \\
& -3 \gamma_{1}\left(B_{1} \bar{B}_{2}+\bar{B}_{1} B_{2}\right)\left(\left|B_{1}\right|^{2}+\left|B_{2}\right|^{2}+2\left|A_{1}\right|^{2}+2\left|A_{2}\right|^{2}\right) \\
& -\frac{3}{2} \gamma_{2}\left(B_{1}^{2} \bar{B}_{2}^{2}+\text { c.c. }\right) .
\end{aligned}
$$

The equations for the modes $A_{\imath}$, (resp. $B_{\imath}$ ), are now obtained from the general equation $\partial w / \partial t=-\delta \mathcal{F} / \delta w$ after multiplication by $\mathrm{e}^{-\imath \mathbf{k}_{A_{\imath}} \mathbf{x}},\left(\right.$ resp. $\mathrm{e}^{-\imath \mathbf{k}_{B_{\imath}} \mathbf{x}}$ ) and integration over the domain.

We get

$$
\begin{aligned}
-\frac{\delta F}{\delta \bar{B}_{1}} & =\frac{\partial B_{1}}{\partial t}+\gamma_{1} \frac{\partial B_{2}}{\partial t} \\
-\frac{\delta F}{\delta \bar{B}_{2}} & =\frac{\partial B_{2}}{\partial t}+\gamma_{1} \frac{\partial B_{1}}{\partial t} \\
-\frac{\delta F}{\delta \bar{A}_{\imath}} & =\frac{\partial A_{2}}{\partial t}
\end{aligned}
$$

and, after inversion,

$$
\begin{aligned}
\frac{\mathrm{d} A_{1}}{\mathrm{~d} t}= & \sigma_{a} A_{1}-6 A_{1}\left(\left|A_{1}\right|^{2}+\left|A_{2}\right|^{2}+\left|B_{1}\right|^{2}+\left|B_{2}\right|^{2}\right)+3\left|A_{1}\right|^{2} A_{1}-6 \bar{A}_{2} B_{1} B_{2} \\
& -3 \gamma_{1} \bar{A}_{2}\left(B_{1}^{2}+B_{2}^{2}\right)-6 \gamma_{1} A_{1}\left(B_{1} \bar{B}_{2}+\text { c.c. }\right) \\
\frac{\mathrm{d} B_{1}}{\mathrm{~d} t}= & \sigma_{b} B_{1}-6 B_{1}\left(\left|A_{1}\right|^{2}+\left|A_{2}\right|^{2}+\left|B_{1}\right|^{2}+\left|B_{2}\right|^{2}\right)+3\left|B_{1}\right|^{2} B_{1}-6 A_{1} A_{2} \bar{B}_{2} \\
& -3 \gamma_{1}\left(\frac{1-\gamma_{2}}{1-\gamma_{1}^{2}}\right) B_{1}^{2} \bar{B}_{2}-3\left(\frac{\gamma_{2}-\gamma_{1}^{2}}{1-\gamma_{1}^{2}}\right) B_{2}^{2} \bar{B}_{1} .
\end{aligned}
$$

The equations for the $A, C$ modes are exactly recovered when $K^{-} \rightarrow 0$, in which case $b$ identifies with $c / 2$ and, $\gamma_{1} \approx 1-(K L)^{2} / 3, \gamma_{2} \approx 1-(2 / 3)(K L)^{2}$ (for the first determination of the detuning parameter) As soon as $R>R_{b}=3\left(1-k_{b}^{2}\right)^{2}$, there are stable steady solutions of this system, involving both $A$ 's and both $B$ 's. For $K=0,2 \phi_{c}-\phi_{a_{1}}-\phi_{a_{2}}=\pi$ and $a^{2}=\left(1-k_{0}^{2}\right) / 3$, 
$c^{2}=R / 3-\left(1-k_{0}^{2}\right)^{2}$, while for $\gamma_{1}=\gamma_{2}=0, \phi_{b}-\phi_{a}=\pi / 2$ and $a^{2}=(1 / 5)(R / 3+(2 / 3)$ $\left.\left(1-k_{b}^{2}\right)^{2}\right), b^{2}=(1 / 5)\left(R / 3-\left(1-k_{b}^{2}\right)^{2}\right)$. The total pattern reconstructed from the first solution corresponds to the undulating rolls already studied by Busse and Auer [17] in the context of the Newell-Whitehead-Segel equation. The second solution describes zig-zags whose wavelength in the $y$ direction is inversely proportional to $\left|\mathbf{k}_{A_{1}}-\mathbf{k}_{B_{1}}\right|$. In general we have $b^{2}=\left(1+\gamma_{1}\right)$ $\left(\sigma_{b}-\left(2 \sigma_{a} / 3\right)\right) /\left(5+4 \gamma_{1}-4 \gamma_{1}^{2}+3 \gamma_{2}\right)$ and $a^{2}=\sigma_{a} / 9-(2 / 3)\left(1+\gamma_{1}\right) b^{2}$ and the free energy reads

$$
F=-\left(2 \sigma_{a} a^{2}+2\left(1+\gamma_{1}\right) \sigma_{b} b^{2}-9\left(a^{4}+b^{4}\right)-12\left(1+\gamma_{1}\right) a^{2} b^{2}-3\left(4 \gamma_{1}+\gamma_{2}\right) b^{4}\right) .
$$

This energy depends on $k_{0}, R, k_{b}$ and $L$ or $\alpha$ which govern the sharpness of the resonance. Note that $\alpha$ has to be proportional to $1 / L$, because the width of the envelope around each quantized mode must at least correspond to the largest scale in the system. It is now interesting to discuss the variation of $F$ as $k_{b}$ varies, for various values of $R$.

For a given $k_{0}$, and $R$ close to $R_{\mathrm{c}}=3\left(1-k_{0}^{2}\right)^{2}$, the free energy of the system decreases monotonically as $k_{b}$ increases from $k_{0}$, except when $L$ is large, in which case there is a small region near $k_{b}=k_{0}$ where the free energy starts increasing first. The energy of the system $(A, C)$ is larger than the one where $\left(B_{1}, B_{2}\right)$ are spaced, indicating a preference for the system to select longer zig-zags rather that sharp undulating rolls.

For the same $k_{0}$ but a value of $R$ larger than a critical value $R_{1}>R_{c}$, one notices a new behavior. The free energy of the solution $\left(A_{1}, A_{2}, C\right)$ is a local minimum, at least when viewed in the sub-space spanned by the five modes $\left(A_{1}, A_{2}, B_{1}, B_{2}, C\right)$. As the modes $B_{1}, B_{2}$ separate symmetrically from $C$, the free energy first rises and then decreases as before but never goes below that of the $\left(A_{1}, A_{2}, C\right)$ solutions. It would appear that the $\left(A_{1}, A_{2}, C\right)$ solution, involving sharply undulating rolls, might actually be stable. This indeed was the man point of the Busse-Auer [17] paper. However, in order to test true stability, we must check against all possible disturbances and expand the configuration space by including many wavevectors in the vicinity of $\mathbf{k}_{A_{1}}, \mathbf{k}_{A_{2}}$ and $\mathbf{k}_{C}$ by using an envelope description. It appears that the $\left(A_{1}, A_{2}, C\right)$ solution is never stable in an infinite geometry. Indeed, as detailed in Appendix $\mathrm{A}$, the condition under which the undulating roll solution $\left(A_{1}, A_{2}, C\right)$ is unstable regarding purely transverse perturbation, is $R<\frac{3-17 k_{0}^{2}}{1-3 k_{0}^{2}}\left(1-k_{0}^{2}\right)^{2}$, whereas it is unstable towards longitudinal (zig-zag) perturbations as soon as $R>7\left(1-k_{0}^{2}\right)^{2}$. leaving no possible stable band. The finite time development of the former instability would appear to lead to domains of straight rolls alternating with domains of undulating rolls, as discovered by Busse and Auer [1]].

2.3. Evolution of ZIG-ZaGs. - From the above discussion, we can now understand that the evolution of the zig-zag instability will depend strongly on the value of the stress parameter. i) If $R$ is smaller than $R_{\mathrm{c}}=3\left(1-k_{0}^{2}\right)^{2}$, a given straight roll pattern whose far field wavenumber is initially $k_{0}<1$, will develop undulations, but since these do not correspond to a stable solution of equations (10), (11), the system will evolve, on an amplitude time scale, by developing reconnections with neighboring rolls such as to bring its modes closer to the critical circle. This has been confirmed numerically by solving the S.H. equations either starting with a set of undulating rolls obtained at $R=097$, taking a smaller value of the stress parameter, $R=0.2$, or starting directly with zig-zag unstable rolls at values of $R$ smaller than $R_{\mathrm{c}}$. Figure 6 shows examples of these situations after many reconnections, where we see mainly straight rolls and dislocations. Note that in the direct simulations of the S.H. equation, the observed value of $R_{\mathrm{c}}$, at which undulating rolls become stable, appears to be slightly larger than the predicted value $R_{\mathrm{c}}=3\left(1-k_{0}^{2}\right)^{2}$.

ii) On the other hand, for $R>R_{\mathrm{c}}$, the system will first settle (on the amplitude fluctuations time scale) on the $\left(A_{1}, A_{2}, C\right)$ solution, which is stable regarding amplitude perturbations. 

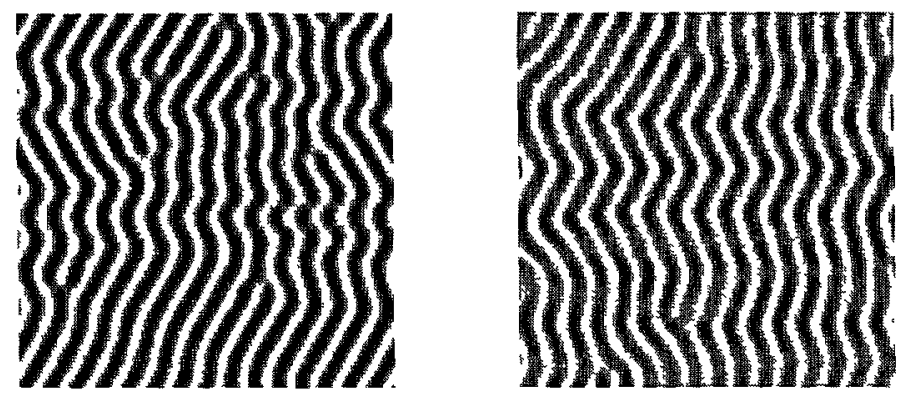

Fig 6 - Transient obtained from a zig-zag instability of straight rolls at $k_{0}=0.9$ and $R=0.2$ (a: left), and restart from the state of Figure 3 (a: right) settıng $R=02$ after $t=2000$

This solution is however unstable regarding phase perturbations and further evolution of the zig-zag pattern will now take place on an horizontal diffusion time scale $\epsilon^{-2}$ and, depending on the stress parameter and external perturbations, can evolve to longer and longer zig-zags modeled by the $\left(A_{1}, A_{2}, B_{1}, B_{2}\right)$ solution. Th1s evolution can take place on time scales even longer than the horizontal diffusion time. This results from the properties of the Cahn-Hilliard equation

$$
\partial_{t} g+\delta g_{y y}+g_{y y y y}-2\left(g^{3}\right)_{y y}=0
$$

which describes the phase dynamics for one dimensional perturbation in the $y$-direction $[8,9]$. We have $g=\theta_{y}$, the $y$ projection of the wavevector and $\delta$ denotes the distance from the zig-zag boundary. This equation admits two kınds of solutions, periodic waves and kinks connecting the states $g_{0}= \pm \sqrt{\delta / 2}$. All periodic solutions are unstable [18] A solution with a given periodicity evolves towards another of longer wavelength. Isolated kinks are stable, but an array of kinks (a situation that obtains when the wavelength of the periodic solution is large) also undergoes coarsening. The difference now is that the interaction between two kinks is exponentally decaying with the distance between them and thus the time it requires to eliminate a pair of kinks is also exponentially long. Because the interaction is so weak, very small external influences can act to pin these "unstable" configurations. This kind of dynamics is very analogous to spin glasses or ordinary glass.

iii) For $R_{c}>R>R_{b}=3\left(1-k_{b}^{2}\right)^{2}$, it is possible to estimate the minimum correlation length of a pattern (defined by $l$, the distance between phase grain boundaries). First of all, let us denote by $\alpha$ the complementary half angle of a zig-zag. or the angle that $\mathbf{k}_{A_{1}}$ makes with the $k_{x}$-axis in Fourier space, in the $\left(A_{1}, A_{2}, B_{1}, B_{2}\right)$ solution (see Fig. 7). The wavelength of the zig-zag in the $y$-direction is inversely proportional to $\left|\mathbf{k}_{A_{1}}-\mathbf{k}_{B_{1}}\right|=\sin \alpha-\sqrt{k_{b}^{2}-\cos ^{2} \alpha}$. For a given value of $R$ greater than $R_{b}$ but smaller than $R_{\mathrm{c}}=3\left(1-k_{c}^{2}\right)^{2}=3 \sin ^{4} \alpha$, value at which the solution $\left(A_{1}, A_{2}, C\right.$ ) starts to exist, it is possible to observe zig-zags (a solution $\left.\left(A_{1}, A_{2}, B_{1}, B_{2}\right)\right)$ whose correlation length is larger than

$$
l_{0}=\frac{1}{\cos \alpha\left(\sin \alpha-\sqrt{\sin ^{2} \alpha-\sqrt{R / 3}}\right)} .
$$

For $\sqrt{R} \ll \sqrt{3} \sin ^{2} \alpha, l_{0} \simeq(2 \sqrt{3} \tan \alpha) / \sqrt{R}$. We thus expect the correlation length $l$ to scale as $R^{-05}$. Note that in experiments of spiral defect chaos [13], the pattern correlation length $\xi$ is found to scale with the deviation $R$ from the critical Rayleigh number as $R^{-043}$. 


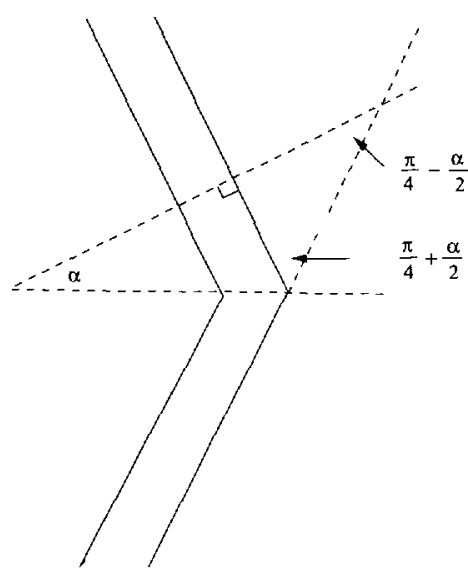

(a)

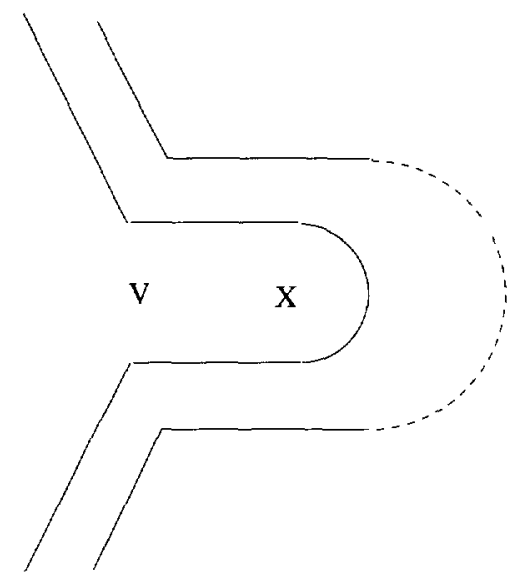

(b)

F1g 7 - Sketch of a "knee" solutions ( $\mathrm{a}$ left) and formation of a VX pair when the angle of this phase grain boundary is too large (b right)

iv) When $R$ exceeds $R_{1}$, the value at which the free energy of the $\left(A_{1}, A_{2}, C\right)$ solution is smaller than that of any of the $\left(A_{1}, A_{2}, B_{1}, B_{2}\right)$ solutions, it is possible to observed "packed" grain boundaries, namely, if defect pinning or topological obstruction (in part due to boundary conditions) occurs, that can stabilize the pattern with respect to phase instabilities. In some cases even, defects are preferred as they can lower the free energy of the system. This is the case of a phase grain boundary whose angle $\alpha$ is too large. Using the regularized C.N. phase equation, we will see in the next Section, that the free energy of the "knee" solution is higher than that of a loop (VX pair) when $\alpha$ exceeds about 35 degrees. Therefore strong zig-zags can help nucleate lamellar patterns (see [1]). Thus, in any physical situation where pinning will occur, the pattern will not eventually relax towards perfectly straight rolls at $k=1$, as the time-dependent phase dynamics would demand, but will instead lock onto a quasi-stationary pattern consisting of patches of straight rolls with $k$ very close to 1 , separated by phase grain boundaries that meet at defects. What is remarkable is that one finds weak and singular solutions of the phase diffusion equation which describe these configurations

2.4. Other Multi-Mode Solutions - Before we explore these situations, it interesting to go back to the mode analysis in order to look for possible multı-mode solutions, i.e. solutions with more than two Fourier modes on the critical curcle. If we project the S.H. equation on two sets of $n$ regularly spaced modes, $A_{2}$ and $B_{\imath}$, lying on two half circles (there is Hermitian symmetry), whose wavenumbers are such that $\left|\mathbf{k}_{A_{2}}\right|=1 .\left|\mathbf{k}_{B_{2}}\right|=\cos (\pi / 2 n)$ and such that the modes on the two circles have the same resonant interaction as described previously, we get:

$$
\begin{aligned}
\frac{\mathrm{d} A_{\imath}}{\mathrm{d} t}= & \sigma_{a} A_{\imath}-6 A_{\imath} \sum_{\jmath=1, n}\left(\left|A_{\jmath}\right|^{2}+\left|B_{\jmath}\right|^{2}\right)+3 A_{\imath}^{2} \bar{A}_{\imath} \\
& -3 \bar{A}_{\imath+1} B_{\imath}^{2}-3 \bar{A}_{\imath-1} B_{\imath-1}^{2} \\
\frac{\mathrm{d} B_{\imath}}{\mathrm{d} t}= & \sigma_{b} B_{\imath}-6 B_{\imath} \sum_{\jmath=1, n}\left(\left|A_{\jmath}\right|^{2}+\left|B_{\jmath}\right|^{2}\right)+3 B_{\imath}^{2} \bar{B}_{\imath}-6 \bar{B}_{\imath} A_{\imath} A_{\imath+1}
\end{aligned}
$$




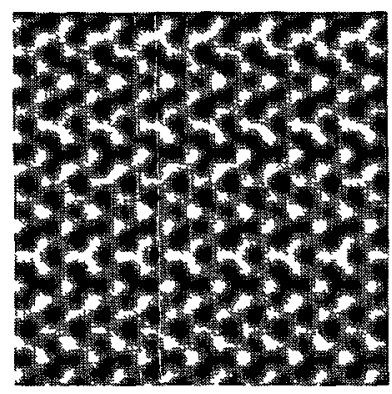

Fig. 8 - Reconstructed pattern obtained from the solution of the dynamical system presented in Appendix B, displaying an array of packed concave disclinations

where $\sigma_{a}$ and $\sigma_{b}$ are the linear growth rates of the $A$ and $B$ modes respectively. This set of equations has been solved numerically and only the cases analogous to the zig-zags, previously described, have been observed.

If, on the other hand, we put six modes on the circle, 60 degrees apart and put the other six $B$-modes on the circle $k=1 / \sqrt{(3)}$ shifted by 30 degrees with respect to the $A$-modes, there is an additional resonant interaction that leads to a solution with non-zero amplitude for every mode. This situation corresponds to an array of packed "concave disclinations" and not hexagons (see Fig. 8). The equations are described in Appendix B.

As a conclusion to this Section, let us mention another experiment supporting the idea that there is a link between the stability of labyrinthic patterns and the width of the band of excited wavenumbers, as distinct from the role of the $k=0$ mode. It consists in solving the quintic and septic S.H equations (where the cubic term $u^{3}$ is replaced by $u^{5}$ and $u^{7}$ respectively). At a given value of the stress parameter, the solutions of the quintıc and septic equations have a much broader spectrum than the usual S.H. equation, due to a weaker nonlinear damping. We find that at $R=0.6$ (for the quintic equation) and at $R=04$ (for the septic equation), patterns that emerge from random initial conditions, look labyrinthic.

\section{Lamellar Patterns and the Phase Equation}

In this section, we propose a description of stationary lamellar patterns containing a low density of defects, based on the phase diffusion equation. We will consider first the phase diffusion equation without regularizing terms and then the case where this regularization is added.

3.1. Stationary Phase Diffusion Equation without Regularization. - Whereas it is clear that the phase equation applies within smooth patches, the description of line and point defects withm the framework of the phase equation, will involve singularities in the wavevector field. Elementary defects are convex $(\mathrm{X})$ and concave $(\mathrm{V})$ disclinations, and grain boundaries which are curves which join disclinations and support discontinuities in roll direction. We will now show that these objects and their composites are exact solutions of the stationary CrossNewell (CN) phase diffusion equation [8], asymptotically valid for slowly modulated patterns in the large aspect ratio limit. Therefore, the theory we present is capable of treating the smooth and singular behavior of the patterns with the same macroscopic equation, and in particular takes account of the restrictions in wavenumber that stable defects must obey as well as defect topology. In these respects, it is much more than a straightforward transfer of the homotopy 
ideas of Toulouse and Kleman, Volovik and Mineyev and Mermin [19] from defects in ordered media to defects in patterns.

We are now going to discuss some solutions of the stationary phase diffusion equation,

$$
\nabla \cdot \mathbf{k} B(k)=0, \nabla \times \mathbf{k}=0
$$

where $\mathbf{k}$ is only determined up to sign. Here we sketch the main ideas. Details will be given elsewhere [20]. Express $\mathbf{x}(x=r \cos \alpha, y=r \sin \alpha)$ as function of $\mathbf{k}(f=k \cos \phi, g=k \sin \phi)$ via the hodograph transformation whence (17), with $\mathbf{x}=\nabla_{\mathbf{k}} \hat{\theta}$, becomes a separable, linear equation $k\left(k B \hat{\theta}_{k}\right)_{k}+(k B)_{k} \hat{\theta}_{\phi \phi}=0$. The phases $\theta(x, y)$ and $\hat{\theta}(f, g)$ are related via the Legendre transformation $\theta+\hat{\theta}=\mathbf{k} \cdot \mathbf{x}=k \hat{\theta}_{k}$. Hence, when we solve for $\hat{\theta}$, we have $\mathbf{x}$ expressed as a function of $\mathbf{k}$ and the constant phase contours can be drawn in both $\mathbf{x}$ and $\mathbf{k}$ space. In general, the map $\mathbf{x} \rightarrow \mathbf{k}$ will be multivalued and the projection of the constant phase contours on the $x, y$ plane will give rise to canonical singularities [20]. For the disclinations $\mathrm{V}$ and $\mathrm{X}$, the double valuedness of $\mathbf{x}$ in $\phi$ means that $\mathbf{k}$ is a director field for which only the single invariant, twist $\mathrm{T}$, the amount by which $\mathrm{k}$ rotates as the singularity is circumscribed on a contour $\mathrm{C}$ in a counterclockwise direction, is defined. For the composite defects such as foci or dislocations, $\mathbf{k}$ will be a vector field for which the additional invariant of circulation $\Gamma=\int_{C} \mathbf{k} \cdot d \mathbf{x}$ is defined.

There are important consequences of the ambiguity of the sign of $\mathbf{k}$. Isolated disclinations are singularities of director fields associated with real order parameters $W=A \cos (\theta / \epsilon)$ ( $A$ the slaved amplitude, $\theta$ the free phase; $\nabla \theta$ is determined only up to sign). As the stress parameter $R$ is reduced to within order $\epsilon^{2}$ of onset, the order parameter becomes complex (both $A$ and $\theta$ are free) and $\nabla \theta$ is determined uniquely from $\nabla W, W=A \exp \imath \theta / \epsilon$. We can thus expect that, close to onset, disclinations cannot survive. Similarly to zig-zags, which were shown to be unstable regarding amplitude perturbations when $R<R_{\mathrm{c}}$, concave and convex disclinations disappear, leading to patterns containing sets of rolls separated by amplitude grain boundaries (numerical evidence is given in [5,9]). Far from onset, the complex S.H. equation (where $u$ is now complex and $u^{3}$ is replaced by $|u|^{2} u$ ) supports however another kind of convex and concave disclination. In Figure $9 \mathrm{a}$ we show the outcome of a numerical simulation of the complex S H. equation, in conditions similar to the ones of Figure 4. A line of dislocations forms in the neighborhood of disclinations (see Fig. 9c for a plot of the squared amplitude $A^{2}=\Re(u)^{2}+\Im(u)^{2}$ ), that has the net effect of creating a cut across which the wavevector changes sign, thus allowing a twist This is not a phase grain boundary but rather an amplitude grain boundary, where shock jump conditions are satisfied (see below). As the stress parameter is reduced to onset (here $R=0.2$ ), these dislocations widen as can be seen from the plot of the amplitude (Fig. 9c) and evolve such as to create the usual grain boundaries where rolls meet at 90 degree angles (see Fig. 9b).

The nature of disclinations is often discussed in terms of harmonic theory ((17) is CauchyRiemann when $B$ is constant) for which $f-\imath g=w$ is $\beta(z-a)^{-1 / 2}, \beta(z-a)^{1 / 2}$, for $\mathrm{X}$ and $\mathrm{V}$ respectively. These solutions, valid for $B$ constant, carry the correct topologies but are irrelevant energetically because $k \rightarrow \infty, 0$ respectively at the defect cores. The correct solutions do have branches which resemble the harmonic solutions and all branches carry the relevant topology and correct twist indices. The harmonic representations, nevertheless, such as $\beta\left(z^{2}-a^{2}\right)^{-1 / 2}\left(\beta=\imath\right.$, vortex; $\beta=1$, target; $\beta$ complex, spiral), $\beta\left(z^{2}-a^{2}\right)^{1 / 2}$ (saddle), $\beta \sqrt{\frac{\left(z+a^{*}\right)(z-a)}{\left(z+b^{*}\right)(z-b)}}\left(\beta=k_{\mathrm{B}}, a=-\imath / k_{\mathrm{B}}+\mu, b=\mu\right)$ (dislocation, in the limits $a, \mu \rightarrow 0$ ). $\beta\left(\frac{z-a}{z+a}\right)^{1 / 2}, \beta=\imath$, type I handle, $\beta=1$ type II handle), help bring out the morphology of defects. One new feature of the present theory is that the map from $\mathbf{x}$ to the wavevector $\mathbf{k}$ is also multivalued due to the nontrivial shape of $k B$. The appropriate branch is chosen from 

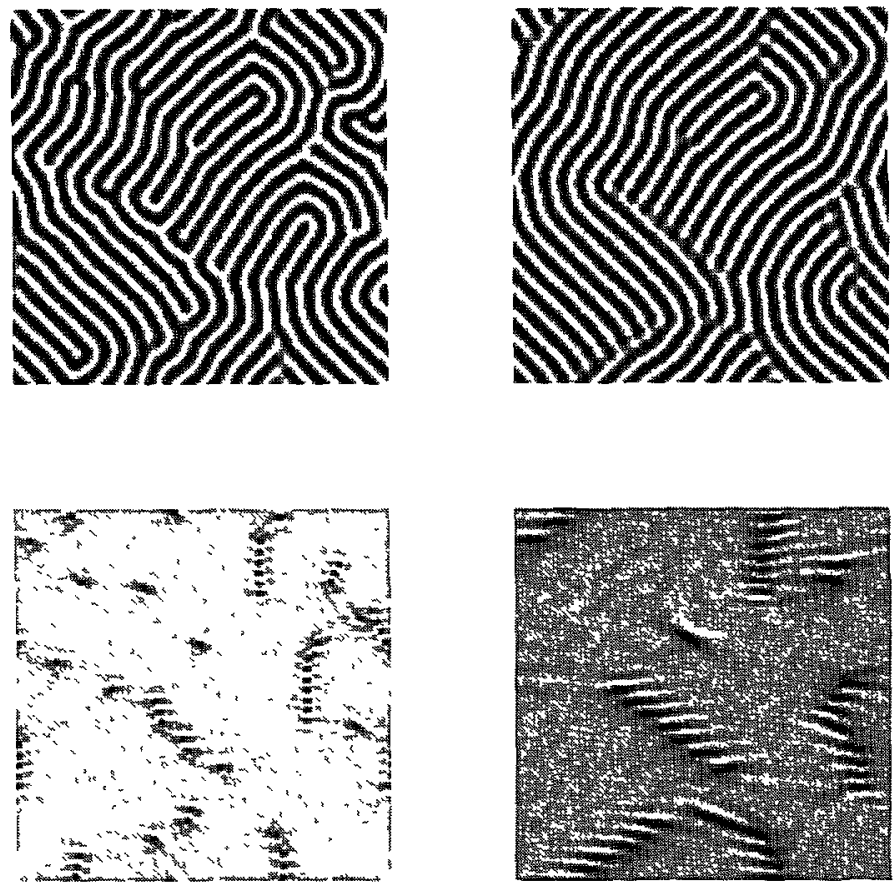

Fig. 9. - Solution of the complex S H. obtaned from a zig-zag instability $\left(k_{0}=0 . \bar{\tau}\right)$ of straight rolls at $R=0.97$ (a left) and $R=02$ (b. right) The amplitude of these patterns is displayed below ((6c) and $(6 \mathrm{~d}))$

stability (energetic) considerations. It turns out that except at the cores of defects and along the phase grain boundaries, $k$ is exponentially close to $k_{\mathrm{B}}$.

We begin with the convex disclination. The exact solution $\hat{\theta}=c\left(k \int_{k_{\mathrm{E}}}^{k} \frac{\mathrm{d} k}{k^{3} B}\right) \cos \phi$ gives $\bar{x}=c\left(\int \frac{d k}{k^{3} B}+\frac{1}{k^{2} B} \cos ^{2} \phi\right), y=c \frac{1}{k^{2} B} \cos \phi \sin \phi$. The twist $T$ is $\pi$. The Roman Arch shape of $\mathrm{X}$ (F1g. 10a) can be calculated explicitly or argued as follows. For $\cos \phi=O(1)$, $\pi / 2>\phi>-\pi / 2$, the $B^{-1} \simeq\left(k-k_{\mathrm{B}}\right)^{-1}$ term dominates the logarithm term $\ln \left|k-k_{\mathrm{B}}\right|$ and $r \simeq \frac{c}{k^{2} B} \cos \phi . \alpha=\phi$. The constant $\theta$ contours are semicircles. However, when $\cos \phi \simeq O(k-$ $k_{\mathrm{B}}$ ), the logarithm singularity dominates and for $x<0, \phi \simeq \pi / 2$ and $k=k_{\mathrm{B}}+\exp \frac{k_{\mathrm{B}}^{3}\left|B^{\prime}\left(k_{\mathrm{B}}\right)\right|}{c} x$, and the $\theta$ contours are straight lines. In order that $k_{\mathrm{B}}<k<k_{\mathrm{E}}$ everywhere down to the disclination core $(r=O(\epsilon))$ where the amplitude regularization comes into play, we must choose $c=-\epsilon c_{0}$. This means that away from the core, $k$ goes exponentially towards $k_{\mathrm{B}}$ Defect parrs XX are given by $\hat{\theta}=-\epsilon c_{0} \int_{k_{\mathrm{E}}}^{k} \frac{\mathrm{d} k}{k B}+\epsilon c_{1} \phi$ and the different manifestations $\left(c_{1}=0\right.$, target; $c_{0}=0, c_{1}=1$, vortex (Fig. 10b), $c \neq 0, c_{1}=1$, spıral) arise from different directions of merger. Each has twist $T=2 \pi$. $\Gamma$ (target) $=0, \Gamma$ (vortex) $=-2 \pi$. All can be regularized in their cores. For $k_{\mathrm{B}} \neq 0\left(k_{\mathrm{B}}=0\right.$ in some models e.g. the CGL equation), isolated vortices cannot exist. When compounded with saddles (see below) they become dislocations.

Solutions for $\mathrm{V}$ (Fig. 10c) can likewise be constructed in the form $\hat{\theta}=F(k) \cos 3 \phi$. The 

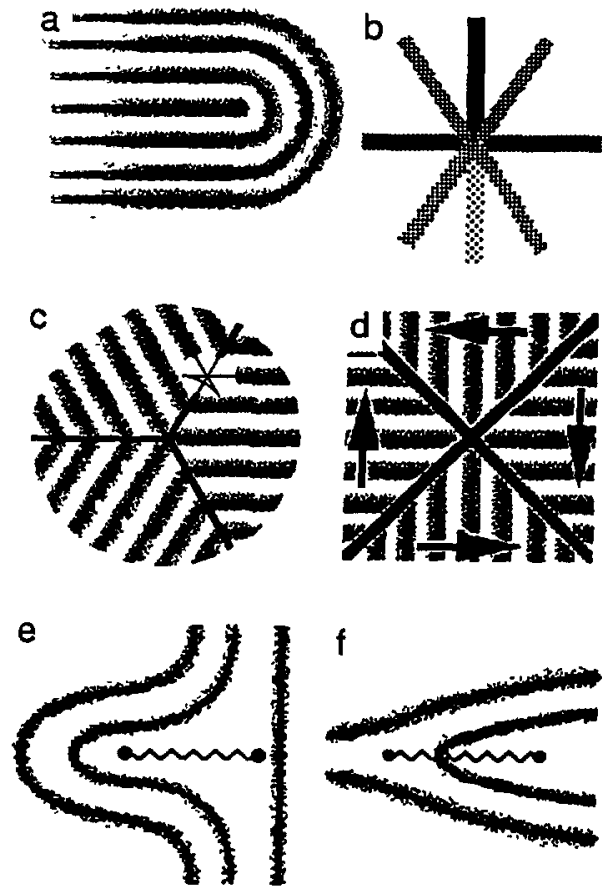

Fig. 10 - a) Convex disclination b) Vortex. c) Concave disclination. d) Saddle. e) Type I handle. f) Type II handle.

function $F$ is the solution of a second order O.D.E. with regular singular points at $k=k_{1}, k=$ $k_{\mathrm{B}}, k=k_{\mathrm{r}}$ and $k=\infty$. On the energetically acceptable branch, $F(k) \simeq \epsilon c_{0} \ln \left|k-k_{\mathrm{B}}\right|$. Almost everywhere in the three sectors $-\pi / 3<\alpha-(2 \pi / 3) n<\pi / 3,(n=0,1,2), \cos 3 \phi=O\left(k-k_{\mathrm{B}}\right)$ and the director field $\mathbf{k}$ has directions $(\bmod \pi)$ of $\pi / 2, \pi / 6$ and $-\pi / 6$ respectively. In each sector $k$ approaches $k_{\mathrm{B}}$ exponentially fast from below. $k=k_{\mathrm{B}}-\exp \left(\left(k_{\mathrm{B}} / 3 \epsilon c_{0}\right) Y(-\sin 3 \phi)\right)$, $Y=-x \sin \phi+y \cos \phi . T(V)=-\pi$ The VV pair (saddle; Fig. $10 \mathrm{~d}$ ), $T(\mathrm{VV})=-2 \pi, \Gamma=0$, is similarly constructed with $\hat{\theta} \simeq \epsilon c_{0} \ln \left|k-k_{\mathrm{B}}\right| \cos 2 \phi$. It is this object that regularizes the vortex by turning its far field phase contours around so that they are parallel to a common direction and form the dislocation, with zero twist (where $C$ circumscribes both singularities) and $-2 \pi$ circulation. The harmonic representation of dislocations, $w=k_{0}+\imath / z, z=x+\imath y$, or $\theta=k_{0} x-\alpha, f=k_{0}+\frac{y}{x^{2}+y^{2}}, g=\frac{-x}{x^{2}+y^{2}}$, captures their topology, a vortex at $x=y=0$ and a saddle at $x=0, y=-1 / k_{0}$. They are not, as often reported, a merger of a single $\mathrm{V}$ and $\mathrm{X}$ because as $k_{0}$ tends to zero, and the singularities separate, one expects a vortex and not a convex disclination to be left at the core The VX pairs (Figs. 10e, f), which we call handles, are important and have inany similarities to dislocations. (Their far field, however, does not become a region of straight parallel rolls.) They should not be viewed as bound pairs such as saddles and vortices because they leave no trace as their separation goes to zero They are like particle-antiparticle pairs which are created out of the vacuum state when energetically advantageous VX formations occur during the nonlinear development of a zlg-zag instability, as in the case of the magnetically driven ferrofluids-

A distinguishing feature of the weak solutions for the concave disclination (and saddle) 
is the presence of lines of discontinuity emanating from the core which we call phase grain boundaries and across which the phase is continuous but the director field is discontinuous. These discontinuities are simply shocks, solutions of $\nabla \cdot \mathbf{k} B+\epsilon^{2} e \frac{\left|B^{\prime}\left(k_{\mathrm{B}}\right)\right|}{4 k_{\mathrm{B}}} \nabla^{4} \theta=0$ in the limit $\epsilon \rightarrow 0$. Since the latter has flux divergence form, the jump conditions can be calculated directly from (17). We find that, denoting $\mathrm{d} x / \mathrm{d} y=s$ the direction of the shock line. the jump conditions are $[f B]=s[g B], s[f]+[g]=0$ and are independent of the exact form of the regularization. For $k$ almost equal to but slightly less than $k_{\mathrm{B}}$ (in which case $\nabla \cdot \mathbf{k} B$ is hyperbolic), they are that the phase contours meet the shock line at equal angles, exactly what is seen. The projection of the constant phase contours gives rise to cusp-like singularitıes [20] in the neighborhood of the shock shown in the inset of Figure 10c. Regularization gives the Maxwell rule for placing the shock. Concave disclinations are often induced in patterns by roll bending. Since (1) is essentially a gradient flow with free energy $F=\int \bar{F} \mathrm{~d} x \mathrm{~d} y, \bar{F}=-1 / 2 \int_{k_{\mathrm{B}}^{2}}^{k^{2}} B(s) \mathrm{d} s+\frac{\epsilon^{2} e}{8 k_{\mathrm{B}}}\left|B^{\prime}\left(k_{\mathrm{B}}\right)\right|\left(\nabla^{2} \theta\right)^{2}$ and since the roll bending occurs over distances of order $\epsilon$ concentrated around shocks, $F$ grows with area if $k \neq k_{\mathrm{B}}$ almost everywhere and with the length of the phase grain boundaries when $k=k_{\mathrm{B}}$. Hence for $k<k_{\mathrm{B}}$, it is not surprising that the optimal patterns are patches of straight parallel rolls, with $k=k_{\mathrm{B}}$, separated by grain boundaries which meet at concave disclinations, as indeed one observes in Figure 2 of [1].

3.2. The Case with Regularization. - It is now interesting to look in more detail at the effect of the regularizing term. We still consider the case where the only regions in which $k>k_{\mathrm{E}}$ are so small (order one in wavelength scale) that no dislocation singularities requiring amplitude regularization are nucleated. The governing equation is the C.N. phase diffusion equation (1), regularized by terms that express resistance to roll curvature as in an elastic medium. For $k$ in the Busse balloon, $\nabla \cdot \mathbf{k} B$ is of order $\epsilon$, as is $\partial \theta / \partial t$, and $\nabla^{2} \nabla \cdot \mathbf{k}$ of order $\epsilon^{3}$. The interesting behavior occurs when $k$ is $\epsilon$ close to $k_{\mathrm{B}}$ or when $k<k_{\mathrm{B}}$, as then $\nabla \cdot \mathbf{k} B$ is hyperbolic and requires the regularization induced by the $\nabla^{4} \theta$ term When $k$ is $\epsilon$ close to $k_{\mathrm{B}}$, $\nabla \mathbf{k} B$ is of order $\epsilon^{2}$ and along the roll derivatives (representing curvature effects) in $\nabla^{4} \theta$ are of order $\epsilon^{1 / 2}$ such that both $\nabla \cdot \mathrm{k} B$ and $\nabla^{4} \theta$ are of order $\epsilon^{2}$. For $k<k_{\mathrm{B}}$ but $k-k_{\mathrm{B}}$ of order one, the boundary layers, in which $\nabla \cdot \mathbf{k} B$ and $\epsilon^{2} \nabla^{4} \theta$ balance, are much sharper, of thickness $\epsilon$.

Equation (1) may be rewritten as $\alpha^{-1} \tau(k) \partial \theta / \partial t=-\delta F / \delta \theta$ with the free energy

$$
F=\int\left(\epsilon^{2}\left(\nabla^{2} \theta\right)^{2}+G^{2}\right) \mathrm{d} x \mathrm{~d} y
$$

where $G^{2}=\frac{-1}{2 \alpha} \int_{k_{\mathrm{B}}^{2}}^{k^{2}} B(s) \mathrm{d} s$ and $\alpha=\frac{\left|B^{\prime}\left(k_{\mathrm{B}}\right)\right| e}{8 k_{\mathrm{B}}}$.

Near $k_{\mathrm{B}}, G^{2}=\frac{1}{2 \alpha} \frac{\left|B^{\prime}\left(k_{\mathrm{B}}\right)\right|}{2 k_{\mathrm{B}}} \frac{\left(k^{2}-k_{\mathrm{B}}^{2}\right)^{2}}{2}=\left(k^{2}-k_{\mathrm{B}}^{2}\right)^{2}$.

We remark that, as long as no moving dislocations are formed, $F$ decreases with time $[8,9]$. In our case, where dislocations are absent, $G$ tends to zero, i.e. $k$ approaches $k_{\mathrm{B}}$ almost everywhere. In regions where $k$ cannot reach $k_{\mathrm{B}}$, due to topological constraints, the term $\left(\nabla^{2} \theta\right)^{2}$ due to curvature, will be important. Here we examine a class of solutions where there is a balance between the two terms of the free energy. We ask if there is a class of solutions to which (1) relaxes, which are governed by equipartition of free energy, i.e

$$
\epsilon \nabla^{2} \theta=s G, \quad s= \pm 1
$$


The answer is simple. Solutıons of (19) are stationary solutions of (1) whenever $\theta=\theta(x, y)$ is a surface with zero Gaussian curvature. i.e. when

$$
\frac{1}{2}[\theta, \theta]=\theta_{x x} \theta_{y y}-\theta_{x y}^{2}=0 .
$$

We give an outline of the proof. The stationary phase equation, $\delta F=0$, leads to

$$
\epsilon^{2} \nabla^{4} \theta-\nabla_{\mathbf{x}}\left(G \nabla_{\mathbf{k}} G\right)=0 .
$$

Let $\epsilon \nabla^{2} \theta=s G$. Applying $\epsilon \Gamma^{2}$ to this equality leads to

$$
\epsilon^{2} \nabla^{4} \theta=s \epsilon \frac{\partial}{\partial x}\left(\frac{\partial G}{\partial \theta_{x}} \theta_{x x}+\frac{\partial G}{\partial \theta_{y}} \theta_{x y}\right)+s \epsilon \frac{\partial}{\partial y}\left(\frac{\partial G}{\partial \theta_{x}} \theta_{x y}+\frac{\partial G}{\partial \theta_{y}} \theta_{y y}\right) .
$$

Then, using (22) and $G=s \epsilon \nabla^{2} \theta$, we get

$$
\begin{aligned}
\epsilon^{2} \nabla^{4} \theta-\nabla_{\mathbf{x}}\left(G \nabla_{\mathbf{k}} G\right)= & s \epsilon \frac{\partial}{\partial x}\left(\frac{\partial G}{\partial \theta_{x}} \theta_{x x}+\frac{\partial G}{\partial \theta_{y}} \theta_{x y}\right)+s \epsilon \frac{\partial}{\partial y}\left(\frac{\partial G}{\partial \theta_{x}} \theta_{x y}+\frac{\partial G}{\partial \theta_{y}} \theta_{y y}\right) \\
& -s \epsilon \frac{\partial}{\partial x}\left(\left(\theta_{x x}+\theta_{y y}\right) \frac{\partial G}{\partial \theta_{x}}\right)-s \epsilon \frac{\partial}{\partial y}\left(\left(\theta_{x x}+\theta_{y y}\right) \frac{\partial G}{\partial \theta_{y}}\right) \\
= & -s \epsilon\left(\frac{\partial^{2} G}{\partial \theta_{x}^{2}}+\frac{\partial^{2} G}{\partial \theta_{y}^{2}}\right)\left(\theta_{x x} \theta_{y y}-\theta_{x y}^{2}\right) \\
= & -s \epsilon \Gamma_{\mathbf{k}}^{2} G[\theta, \theta] .
\end{aligned}
$$

The result follows, after noting that $\nabla^{2} G$ is bounded and tends to 4 when $k$ goes to $k_{\mathrm{B}}$.

This result is relevant to our problem because (20) holds almost everywhere for some of the most significant stationary solutions as we will show. First, however, we note that when $k$ stays close to $k_{\mathrm{B}}$, and after approximating $G$ by $k_{\mathrm{B}}^{2}-k^{2}$, equation (19) can be linearized. Indeed, setting $\theta=-s \epsilon \log \Psi$, we obtain

$$
\epsilon^{2} \nabla^{2} \Psi-k_{\mathrm{B}}^{2} \Psi=0
$$

To describe solutions to (24) it will be easiest to use the equation in unscaled variables,

$$
\nabla^{2} \Psi-k_{\mathrm{B}}^{2} \Psi=0,
$$

and if necessary introduce the scaling directly in the solutions. For instance, for solutions describing small variations about straight parallel rolls, i.e. $\theta=k_{\mathrm{B}} x+\tilde{\theta}\left(\epsilon x / k_{\mathrm{B}}, \sqrt{\epsilon} y\right)$, we have $\nabla^{4} \theta=O\left(\epsilon^{2}\right)$ and $\nabla \cdot \mathbf{k} B=O\left(\epsilon^{2}\right)$ while $[\theta, \theta]=O\left(\epsilon^{3}\right)$. Noting $\Psi=\mathrm{e}^{-k_{\mathrm{B}} x} f\left(\epsilon x / k_{\mathrm{B}}, \sqrt{\epsilon} y\right)$, we verify that $f$ satisfies the heat equation This is exactly the Hopf-Cole transformation already obtained in [12]. Examples of such solutions are also described in [9].

Solutions to (25) are good approximate solutions, but notice that they do not describe interaction between defects. Its simplest solution describes roll bending through an angle $\pi-2 \alpha$,

$$
\Psi=\mathrm{e}^{-\left(k_{\mathrm{B}} \cos \alpha\right) x} 2 \cosh \left(k_{\mathrm{B}} \sin \alpha\right) y
$$

For that "knee" solution (see Fig 7 , which is a sketch when $\epsilon \rightarrow 0$ ), the Gaussian curvature is exactly zero and $k$ approaches $k_{\mathrm{B}}$ exponentially fast within a distance of a roll wavelength from $y=0$. Thus the linearized equation is a very good approximation except in a very small portion of space. The free energy of the solution (26) per unit distance is (4/3) $k_{\mathrm{B}}^{3} \sin ^{3} \alpha$. This means that if the bend is too large the system will prefer to create a loop (a VX pair), when the latter has a lower free energy, i.e. when $\sin ^{3} \alpha>2 \sin ^{3}(\pi / 4-\alpha / 2)$, which occurs for about $35^{\circ}$. Therefore strong zigzags can help nucleate lamellar patterns. 


\section{Summary}

Lamellar patterns emerging from a cellular instabılity exhıbit a juxtaposition of patches whose typical size $l$ decreases as the value of the stress parameter $R$ of the system increases. Using a modal analysis of simple undulating (and multimode) solutions of the S.H equation, we have argued that, for a given value of $R$, these patches undergo a coarsening on an amplitude (rapid) time scale if their size is smaller than a critical value, and evolve slowly, on a phase diffusion time scale, if their size is larger After some phase adjustments. pinning can occur due to defects and boundary conditions, and the time evolution becomes extremely slow. The critical value of $l$ has been computed for a special solution of undulating rolls (see also [17]). More precisely we have shown that if we fix $l$, the typical wavelength of the undulation, a simple solution exists for $R$ larger than a critical value $R_{c}$. This solution is based on a resonant interaction between Fourier modes lying both on the critical circle $k=1$ and inside this circle. When $R$ is sufficiently large, labyrinthic patterns obtain (when $l$ is of the order of a few roll wavelengths). which look like those observed in bistable systems, but whose formation mechanism is nevertheless very different.

In a second part of this paper we have shown that stationary lamellar patterns are well described by the phase diffusion formalism. Whereas this is not surprising for the slowly varying patches, it is remarkable that defects can also be captured by special solutions of this large scale equation. A lot of questions remain to be addressed in order to provide a complete description of these systems One of the challenges is, for example, to describe the temporal dynamics using the phase diffusion equation

\section{Acknowledgments}

We are thankful to S. Fauve and Y. Pomeau and to the Centre Emile Borel, for their hospitality at the Institut Poincaré, during the Physics semester devoted to "Nonlinear waves", where part of this work has been performed. Useful discussions with A. Nepomnyashchy are gratefully acknowledged. This work was supported by AFOSR900021 and NATO 9.25, 921149.

\section{Appendix A}

In this appendix. we study the long-wavelength instabilities of the undulating roll solution, modeled by three modes of wavevectors $\mathbf{k}_{A_{1}}, \mathbf{k}_{A_{2}}$ and $\mathbf{k}_{C}$ We will consider wavepackets centered around these modes. For generality, and in order to compare with results of Busse and Auer [17], $A_{1}$ and $A_{2}$, supposed to be symmetric about the axis $k_{y}=0$, will not be assumed to lie on the critical circle. The envelope equations, can be written immediately:

$$
\begin{aligned}
\frac{\partial A_{1}}{\partial t}= & \left(\sigma_{a}-2\left(1-k_{a}^{2}\right)\left(2 \imath \mathbf{k}_{A_{1}} \cdot \nabla+\nabla^{2}\right)-\left(2 \imath \mathbf{k}_{A_{1}} \cdot \nabla+\nabla^{2}\right)^{2}\right) A_{1} \\
& -6 A_{1}\left(\left|A_{1}\right|^{2}+\left|A_{2}\right|^{2}+|C|^{2}\right)+3\left|A_{1}\right|^{2} A_{1}-3 \bar{A}_{2} C^{2} \\
\frac{\partial A_{2}}{\partial t}= & \left(\sigma_{a}-2\left(1-k_{a}^{2}\right)\left(2 \imath \mathbf{k}_{A_{2}} \cdot \nabla+\nabla^{2}\right)-\left(2 \imath \mathbf{k}_{A_{2}} \quad \nabla+\Gamma^{2}\right)^{2}\right) A_{2} \\
& -6 A_{2}\left(\left|A_{1}\right|^{2}+\left|A_{2}\right|^{2}+|C|^{2}\right)+3\left|A_{2}\right|^{2} A_{2}-3 \bar{A}_{1} C^{2} \\
\frac{\partial C}{\partial t}= & \left(\sigma_{c}-2\left(1-k_{c}^{2}\right)\left(2 \imath k_{c} \partial_{X}+\Gamma^{2}\right)-\left(2 \imath k_{c} \partial_{X}+\Gamma^{2}\right)^{2}\right) C \\
& -6 C\left(\left|A_{1}\right|^{2}+\left|A_{2}\right|^{2}+|C|^{2}\right)+3|C|^{2} C-6 A_{1} A_{2} \bar{C}
\end{aligned}
$$

The undulating roll solution reads: $A_{1}=A_{2}=a . C=v v$, with $a^{2}=\left(\sigma_{a}-\sigma_{c}\right) / 3$ and $c^{2}=\sigma_{c}-\left(2 \sigma_{a} / 3\right)$ 
Perturbing this solution in the form

$$
\begin{aligned}
A_{1} & =a\left(1+u_{1}\right) \mathrm{e}^{\imath \phi_{1}} \\
A_{2} & =a\left(1+u_{2}\right) \mathrm{e}^{\imath \phi_{2}} \\
C & =\imath c(1+v) \mathrm{e}^{\imath \phi_{c}}
\end{aligned}
$$

the linearized equations read

$$
\begin{aligned}
\frac{\partial u_{1}}{\partial t}= & \sigma_{a} u_{1}+2\left(1-k_{a}^{2}\right)\left(2\left(\mathbf{k}_{A_{1}} \nabla\right) \phi_{1}-\nabla^{2} u_{1}\right)-\left(\nabla^{2}-4\left(\mathbf{k}_{A_{1}} \cdot \nabla\right)^{2}\right) u_{1} \\
& +4\left(\mathbf{k}_{A_{1}} \cdot \nabla\right) \nabla^{2} \phi_{1}-\left(15 a^{2}+6 c^{2}\right) u_{1}-\left(12 a^{2}-3 c^{2}\right) u_{2}-6 c^{2} v \\
\frac{\partial \phi_{1}}{\partial t}= & 3 c^{2}\left(2 \phi_{c}-\phi_{1}-\phi_{2}\right)-2\left(1-k_{a}^{2}\right)\left(2\left(\mathbf{k}_{A_{1}} \cdot \nabla\right) u_{1}+\nabla^{2} \phi_{1}\right) \\
& -4\left(\mathbf{k}_{A_{1}} \cdot \nabla\right) \nabla^{2} u_{1}-\left(\nabla^{2}-4\left(\mathbf{k}_{A_{1}} \cdot \nabla\right)^{2}\right) \phi_{1} \\
\frac{\partial v}{\partial t}= & \sigma_{c} v+2\left(1-k_{c}^{2}\right)\left(2 k_{c} \partial_{X} \phi_{c}-\nabla^{2} v\right)-\left(\nabla^{2}-4 k_{c}^{2} \partial_{X X}\right) v \\
& +4 k_{c} \partial \nabla_{X}^{2} \phi_{c}-6 a^{2}\left(u_{1}+u_{2}\right)-\left(6 a^{2}+9 c^{2}\right) v \\
\frac{\partial \phi_{c}}{\partial t}= & -6 a^{2}\left(2 \phi_{c}-\phi_{1}-\phi_{2}\right)-2\left(1-k_{c}^{2}\right)\left(2 k_{c} \partial_{X} v+\nabla^{2} \phi_{c}\right) \\
& -4 k_{c} \partial_{X} \nabla^{2} v-\left(\nabla^{2}-4 k_{c}^{2} \partial_{X X}\right) \phi_{c}
\end{aligned}
$$

where the equations for $u_{2}, \phi_{2}$ are obtained from those for $u_{1}, \phi_{1}$ by replacement of the index 1 by 2 . The global phase mode is $a^{2}\left(\phi_{1}+\phi_{2}\right)+c^{2} \phi_{c}$. In order to study the stability domain, it is enough to keep terms up to the second order in the phase gradients. After some algebra. we find that the condition for a phase instability in the $X$ direction is

$$
\begin{gathered}
\left(R-3\left(1-k_{c}^{2}\right)^{2}+2\left(1-k_{a}^{2}\right)^{2}\right)\left(1-3 k_{c}^{2}\right) \\
+2\left(k_{a}^{2}-k_{c}^{2}\right)\left(2-k_{c}^{2}-k_{a}^{2}\right)\left(1-k_{a}^{2}-2 k_{c}^{2}\right) \\
+4 k_{c}^{2}\left(1-k_{c}^{2}\right)\left(1-3 k_{c}^{2}+2 k_{a}^{2}\right)+8 k_{c}^{2}\left(1-k_{a}^{2}\right)\left(k_{c}^{2}-k_{a}^{2}\right)>0 .
\end{gathered}
$$

Writing $k_{c}=1+q$ and $k_{a}^{2}=k_{c}^{2}+p^{2}$, with $p$ and $q$ small compared to one, one recovers the results of [17], after noting the rescaling of the space variable and of the amplitude. When $k_{a}=1$, condition $(\mathrm{A} .9)$ reduces to

$$
R<\frac{3-17 k_{c}^{2}}{1-3 k_{c}^{2}}\left(1-k_{c}^{2}\right)^{2}
$$

while the condition for a phase instability along the $Y$ directıon reads

$$
R>7\left(1-k_{c}^{2}\right)^{2} \text {. }
$$

In consequence, there is no stable region.

\section{Appendix B}

In this appendix, we write the equations for the special arrangement of six modes, regularly placed on the critical circle, 60 degrees apart, together with six inner modes located on the circle $k=1 / \sqrt{3}$, shifted by 30 degrees with respect to the first ones. We denote by $A_{1}$, $A_{2}$ and $A_{3}$ the successive modes on the critical circle, the three other ones being given by hermitian symmetry. On the inner circle $B_{\imath}$ denotes the mode located between $A_{\imath}$ and $A_{\imath+1}$. The equations read

$$
\frac{\mathrm{d} A_{1}}{\mathrm{~d} t}=\sigma_{a} A_{1}-6 \Sigma A_{1}+3 A_{1}^{2} \bar{A}_{1}-6 B_{1} B_{2} \bar{A}_{3}-6 \bar{B}_{2} \bar{B}_{3} A_{2}
$$




$$
\begin{aligned}
& -3 B_{1}^{2} \bar{B}_{2}-3 \bar{B}_{3}^{2} B_{2} \\
\frac{\mathrm{d} B_{1}}{\mathrm{~d} t}= & \sigma_{b} B_{1}-6 \Sigma B_{1}+3 B_{1}^{2} \bar{B}_{1}-6 A_{1} A_{3} \bar{B}_{2}-6 A_{2} \bar{A}_{3} B_{3} \\
& -6 \bar{B}_{1} \bar{B}_{3} A_{2}-6 \bar{B}_{1} B_{2} A_{1}-3 B_{2}^{2} \bar{A}_{3}-3 \bar{B}_{3}^{2} A_{3}
\end{aligned}
$$

where $\Sigma=\left|A_{1}\right|^{2}+\left|A_{2}\right|^{2}+\left|A_{3}\right|^{2}+\left|B_{1}\right|^{2}+\left|B_{2}\right|^{2}+\left|B_{3}\right|^{2}$. The other equations are obtained by circular permutation of the indices. Stationary solutions of this system can be found, where all the modes are nonzero. After some simple analysis we find a simple nontrivial solution that read:

$$
\begin{gathered}
A_{1}=a, \quad A_{2}=-a, \quad A_{3}=-\imath a \\
B_{1}=\imath b, \quad B_{2}=b, \quad B_{3}=b
\end{gathered}
$$

with

$$
\begin{array}{r}
R-15 a^{2}-6 b^{2}=0 \\
\sigma_{b}-6 a^{2}-15 b^{2}=0
\end{array}
$$

This solution exists for $R>20 / 27$ and, using numerical simulations, it is found to be stable for $R>1.6$ The reconstructed pattern is shown in Figure 8 where we clearly see an array of concave disclinations.

\section{References}

[1] Seul M., Monar L.R., O'Gorman L. and Wolfe R, Scrence 254 (1991) 1616; Seul M. and Wolfe R., Phys Rev. Lett 68 (1992) 2460

[2] Ouyang Q and Swinney H L., Chaos 1 (1991) 411

[3] Lee K J , McCormick W.D, Ouyang Q. and Swınney H.L., Scrences 261 (1993) 192.

[4] Decker W., Pesch W. and Weber A., Phys. Rev Lett. 73 (1994) 648

[5] Geddes J.. Ph.D Thesis, University of Arızona (1994).

[6] Petrich D.M and Goldstein R.E., Phys Rev. Lett 72 (1994) 1120

[7] Hagberg A and Meron E., Phys Rev Lett 72 (1994) 2494

[8] Cross M C. and Newell A.C, Physıca D 10 (1984) 299

[9] Passot T and Newell A.C, Physaca D 74 (1994) 301.

[10] Pomeau Y., Zaleskı S., Manneville P and Agnew Z., Math Phys. 36 (1985) 367.

[11] Newell A.C, Passot T and Soulı M., J. Flurd Mech 220 (1990) 187.

[12] Nepomnyashichy A, Pismen L, Phys Lett, A 153 (1991) 427

[13] Morris, J Bodenshatz. E., Cannel, D, and Ahlers, G. Phys Rev Lett (1993) 712026

[14] Gunaratne G H., Ouyang Q. and Swinney H L., Pattern formation in the presence of symmetries, to appear in Phys Rev Lett 75 (1995) 834.

[15] Cross M.C and Tu Y., Phys Rev Lett 75 (1995) 834

[16] Frisch T., Newell A.C and Passot T., preprint.

[17] Busse F. and Auer M., Phys Rev Lett 72 (1994) 3178

[18] Kawasaki K and Ohta T., Physıca A 116 (1982) 573.

[19] Toulouse G and Kleman M, J Phys Lett France 37 (1976) L-149; Volovik G.E. and Mineyev V P., Sov. Phys. JETP 45 (1977) 1186, Mermin N.D, Rev Mod. Phys. 51 (1979) 591

[20] Ercolani N., Indik R, Newell A.C., Passot T , in preparation. 
Classification

Physics Abstracts

$05.45 \mathrm{~b}-4755 \mathrm{k}$

\title{
Noise/Chaos Distinction Applied to the Study of a Fluidized Bed
}

\author{
N. Letaief, C. Rozé and G. Gouesbet \\ LESP(*), INSA de Rouen, BP08, 76131 Mont-Saint-Aignan Cedex, France \\ (Received 4 August 1994, reised 1 February 1995, accepted in final form 18 August 1995)
}

\begin{abstract}
The observation of chaos in fluidization have been claimed by some authors We analyze the dynamics of a gas-solid fluidized bed and demonstrate that the dynamics is not chaotic but noisy. The random process is studied by using the Hurst analysis in order to look for long-term correlations in the signals. The dynamics may then be modelled by a fractal Brownian motion process.
\end{abstract}

\section{Introduction}

Though now widely used in industrial processes, fluidized beds are stıll somewhat not understood from a fundamental point of view In particular, fundamental approaches are limited by the complexity of the equations involved, and statistical approaches, which are very useful in practical cases, can only give us approximate values. In recent years, many researchers have taken an interest in the chaotic behavior of fluidization. The reason for this interest is probably in part linked to a fundamental property of chaotic systems, i.e. the main features of the underlying dynamics can be retrieved from a single well-chosen time series. Nevertheless, there is no general agreement between experimenters on the subject: Van Den Bleek and Schouten [1] clam that a fluidization process can be characterized by a low-dimensional strange attractor, but Tam and Devine [2] claim exactly the contrary. Such contradictions may be due to the fact that phenomena grouped under the same vocable "fluidization" can actually take quite different forms (slugging or bubbling, etc...), and, therefore, the presence or absence of chaos may sensitively depend on the actual experiments under study.

But, on the other hand, as emphasized by Provenzale et al. [3], it is now recognized by many experimenters that one method is not sufficient to establish evidence for chaos, and that the simultaneous use of several methods can be required to avoid incorrect conclusions [4]. For instance, as far as the evaluation of correlation dimension is concerned, Rapp et al. [5] showed that filtered noise can seemingly behave as low-dimensional attractors and the same phenomena have been observed by us with an autoregressive random process. Therefore, invoking a measured small correlation dimension to conclude about the existence of low-dimensional chaos may be misleading. With other methods, such as the computations of the Lyapunov

(*) URA CNRS 230-CORIA 


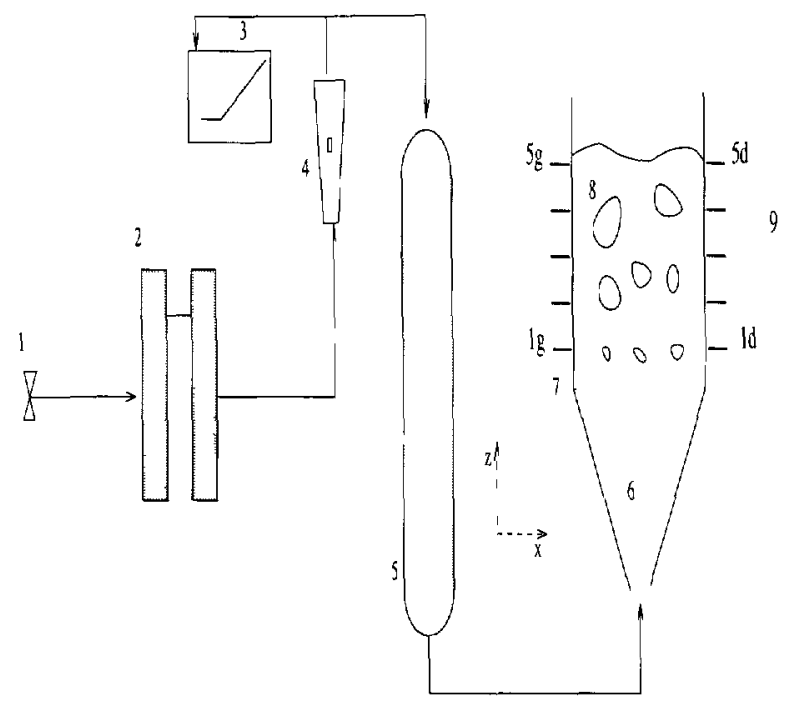

Fig 1 - Experimental set-up. (1) air supply, (2) sub-micronic filters, (3) rotameter, (4) mercury, (5) vacuum chamber, (6) dessication, (7) distributor, (8) fluidization, (9) pressure taps.

exponents (or even of the largest Lyapunov exponent), experimental conditions can prevent the algorithms working, and do not give any interpretable results in many real cases (for which noise is important). Thus, the correct attitude when dealing with problematic data is to examine experimental results by several means. using a battery of tests, before establishing a diagnosis of the chaotic or random nature of the data. Obviously, this heuristic attitude is very different from that in the case of well-known chaotic data.

Our aim. in this paper. is to determine the nature of signals recorded from a fluidized bed, and, in particular, to establish whether our data result from a chaotic process or not. We therefore use several methods of investigation, and among them, the method of surrogate data, discussed by Theiler [6] in 1992. This method allows systematic comparisons between noise and chaos. Using this method as well as other more classical methods, we will conclude that the data from our fludized bed are not the results of a low-dimensional chaotic system.

The paper is organized as follows. In Section 2, the experimental set-up and the basic features of measured signals are presented Section 3 is devoted to the description of noise/chaos distinction methods, and to their application to some well-known benchmark examples Section 4 is devoted to the applications of the methods of distinction between noise and chaos to our particular case. Section 5 describes the Hurst analysis, which is a method of characterization of the long-term behavior of signals, and its application to the experimental data. Section 6 is a conclusion

\section{Description of the Experiments}

2.1. Experimental Set-up. - The set-up is represented in Figure 1. The fluidized bed is two-dimensional: its inner dimensions are $300 \times 500 \times 10 \mathrm{~mm}^{3}$ The walls are in plexiglas, to allow visual observation. The fluidization gas employed is air, filtered and dried beforehand in a dessication column. The parameter is the flow rate of air divided by the distributor area, also called superficial velocity. The distributor is a porous medium made out of bronze, thus 
ensuring a regular distribution of the air flow. The static depth of the particle bed is about $240 \mathrm{~mm}$. The fluidized particles are thin glass balls, their diameter being between 100 and $200 \mu \mathrm{m}$. We thus have a deep bed (by opposition to shallow beds), belonging to the group $\mathrm{B}$ of Geldart's classification.

Two specific values of the superficial velocity are important here: the minimum fluidization velocity $\left(v_{\mathrm{mf}}\right)$, which corresponds to the state where the drag forces balance the weight of the particles, and the minimum bubbling velocity $\left(v_{\mathrm{mb}}\right)$, corresponding to the appearance of the first bubbles. For the group of beds here considered, $v_{\mathrm{mf}}$ is nearly equal to $v_{\mathrm{mb}}(\approx 0.05 \mathrm{~m} / \mathrm{s})$, i.e. we cannot in practice distinguish between the incipient fluidization and the subsequent onset of bubbling instability which appears for an extra very small increase of the control parameter Th1s means that one cannot expect to explicitly observe a road towards chaos. On the contrary a fully developed chaos (or random process) is reached almost immediately.

After their apparition, bubbles grow by coalescence as they rise, this coalescence process having been previously extensively investigated [8].

2.2. MeAsured QuANTITIES - In a first step, bubbling being identified as a fundamental process in the bed, we measured a quantity directly related to this process. Then, we recorded the appearance and evolution of bubbles at a given point of the bed referenced by its coordinates $(x, z)$ (Fig. 2). A laser beam source and a photodetector are placed in front of each other When bubbles pass in front of the detector, the laser beam attenuation is small, and the corresponding photodetector signal is high. Otherwise, it is practically equal to zero, leading to an essentially binary signal, as shown in the figure (after use of a threshold level). From such a signal, we may determine the transit time $t_{\mathrm{p}}$ of bubbles and the delay time $t_{\mathrm{c}}$ between bubbles The whole procedure has been validated by filming the bed, simultaneously recording the photodetector signal, and comparing the characteristic times $t_{\mathrm{p}}$ and $t_{\mathrm{c}}$, measured from both the signal and the film. Here it will suffice to say that the analysis of time series built from successive $t_{\mathrm{p}}$ 's or successive $t_{\mathrm{c}}$ 's leads to the unambiguous conclusion that the dynamics is random without any indication of any deterministic components [9].

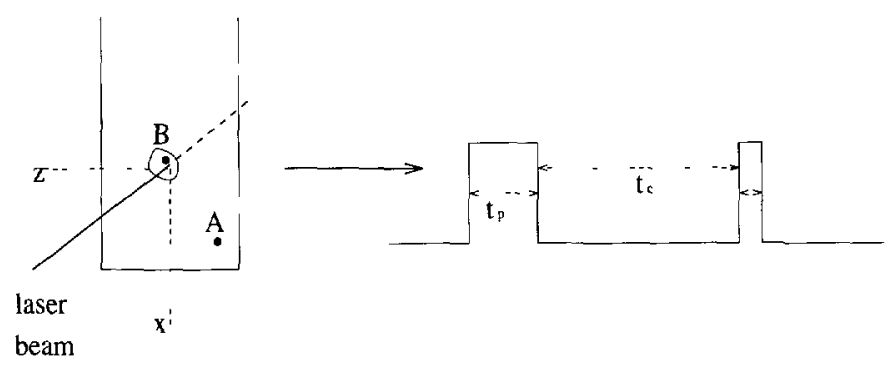

flundized bed

S1gnal in the tıme domain

Fig. 2. - Emergence and vanishing time recordıng of bubbles.

This conclusion should however be softened by considering the following items :

i) Measurements based on bubble characterizations are essentially local and, by introducing some kind of spatial averaging. we may wonder whether a global deterministic component could not be detected. 
ii) Analyzed time series are discrete and thresholdıng may have introduced spurious effects and spoilt some relevant information.

Therefore, it has been decided to measure quantities being both global (i.e. producing some spatial average) and time continuous, namely pressures. Taps have then been inserted laterally on each side along the fluidized bed (Fig. 1), so that either the pressure at a given altitude in the set-up, or the difference of pressure between two given points could be measured. Pressure measurements are carried out by using a membrane pressure sensor, with a precision of $0.2 \%$ and a time response of $10 \mathrm{~ms}$. Signals are sampled and recorded by using a Sun4 SPARC workstation. We observed that there was no significant frequency above $50 \mathrm{~Hz}$ in the pressure dynamics. Therefore, as indicated by Shannon's theorem, sampling frequency should be greater than or equal to $100 \mathrm{~Hz}$. A sampling rate equal to $200 \mathrm{~Hz}$ has then be chosen, producing smooth curves, without any apparent noise at the visual level.

2.3. Experimental Results. - Fan et al [10] have experimentally demonstrated that the pressure fluctuations at a given altitude were due essentially to bubbles crossing at this altitude, the contribution from distant bubbles being less important than that from neighbouring ones. We can thus estimate that the recorded signals correspond to global measurements of the phenomenon, and is representative of the experimental set-up, with a spatial averaging on an horizontal section.

Typical examples of recordings are shown in Figure 3, for two superficial velocities: the smallest one is just a little larger than the experimental minimum of fluidization velocity, and the other corresponds to more than twice that velocity. The only observation possible is the irregularity of the signal, whatever the superficial velocity. The Fourier transforms of the time series are broad-band (Fig. 4), and they are typical of fluidization [11-14]. They also present strong similarities with the spectra obtained for a bubble column [15] (where the particles are replaced by a liquid medium).

One can define a characteristic frequency by averaging all the frequencies corresponding to amplitudes greater than $80 \%$ of the total integrated amplitude of the spectrum. This characteristıc frequency increases with the gas velocity. This is related to the fact that although

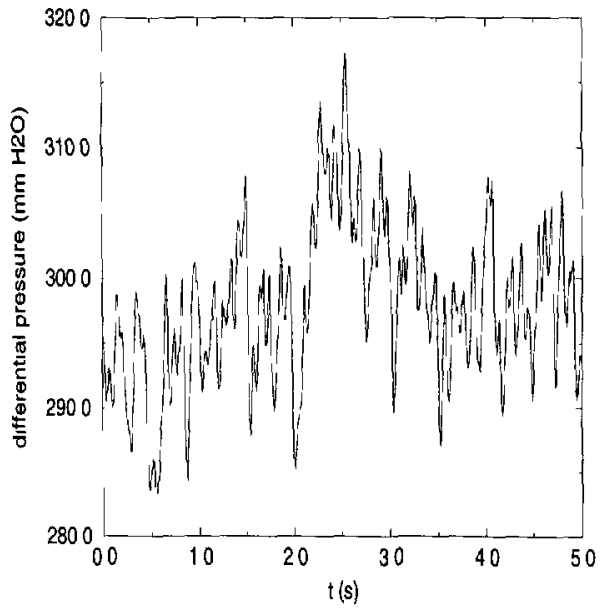

a)

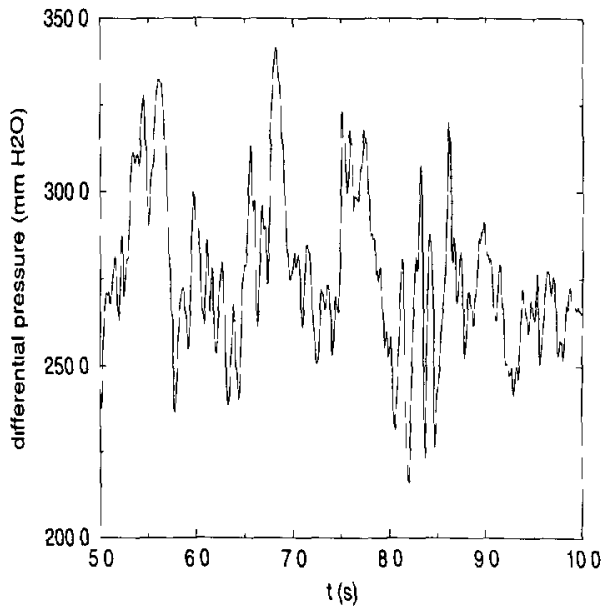

b)

Fig. 3 - Left-hand differential pressure recordings for two superficial velocities. 


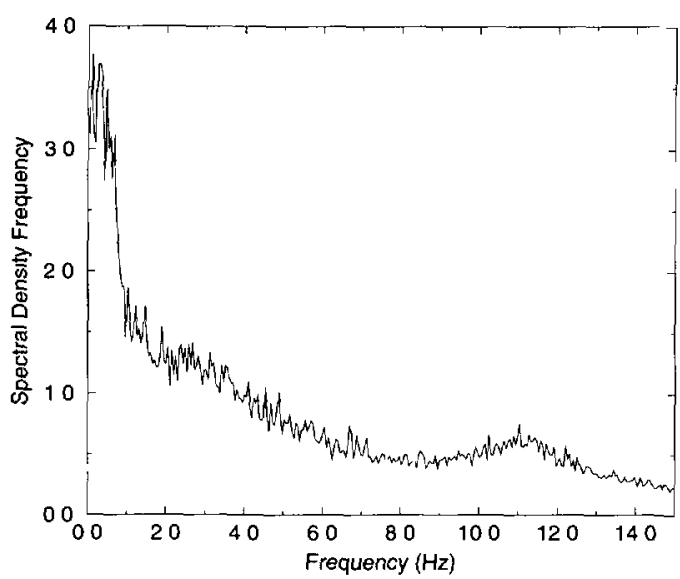

a)

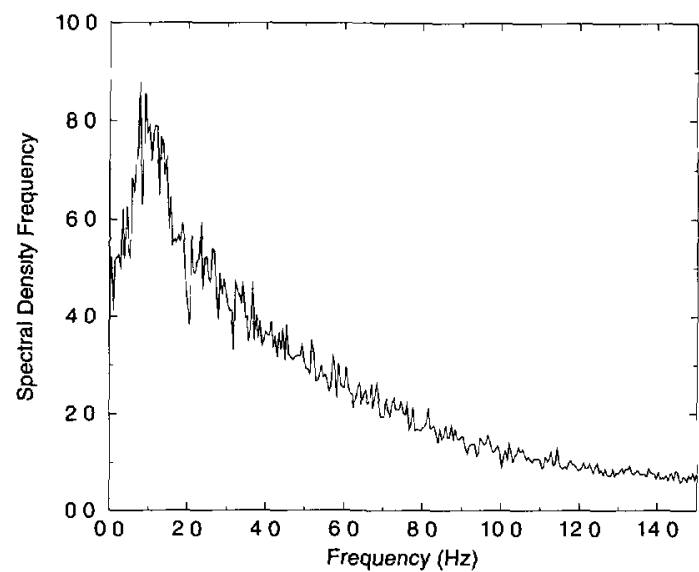

b)

Fig. 4 - Left-hand differential pressure for two superficial velocities: Fourıer's spectra.

bubbles diameters increase with gas velocity, their velocity also increase, eventually leadıng to higher frequencies

From a time series $\left(x_{2}\right)$, we reconstructed a trajectory $\left(X_{2}\right)$ using the method of delays, with a lag time $p$, in a reconstructed phase space of dimension $n$. Presented results correspond to a sampling time of $5 \times 10^{-3} \mathrm{~s}$, to be compared with the inverse of the characteristic frequency which is about $5 \mathrm{~s}$. For the dimension calculations later presented, the sampling time has been varied, i.e. increased up to $2 \times 10^{-2} \mathrm{~s}$ without modifyıng the results. Conversely, a sampling time of $5 \times 10^{-3} \mathrm{~s}$ may be too short to analyze forecasting errors (see beginning of Sect. 4).

In a three-dimensional phase space, the reconstructed trajectory (Fig. 5) is nearly twodimensional The two-dimensional character of the object is actually not apparent in the figure, but becomes evidenced if it is rotated on a screen using a graphical AVS system. We did not detect any perıodic orbits nor other regular structures like recurrences. i.e. the trajectory seems essentially desorganized.

Also, in agreement with the two-dimensional character of the object exhibited in Figure 5, we evaluated correlation dimensions using the Grassberger-Procaccia algorithm (see [16] and many references in [17]), which we found to be equal to about 2 . This fact is however not sufficient to conclude to the presence of a low-dimensional attractor. In particular, we reproduced similar results by using the Grassberger-Procaccia algorithm on a time series generated by autoregressive noise. Therefore, a stochastic process analyzed by evaluating correlation dimensions may lead to the spurious identification of deterministic chaos. Such a conclusion has previously been also pointed out by Rapp et al. [5] by analyzing time series generated by a filtered noise. Due to such a fact, it is likely that some claims about the presence of chaos in experimental sıgnals, includıng fluidization, could be incorrect. In some cases, a most reliable conclusion must therefore be obtained by using noise/chaos distinction techniques exposed in the next section.

\section{Noise/Chaos Distinction Methods}

Two techniques are discussed in this section. The first one, relying on the discussion of forecasting errors, has been developed by Farmer and Sidorowıch [18] and was used by Sugihara 


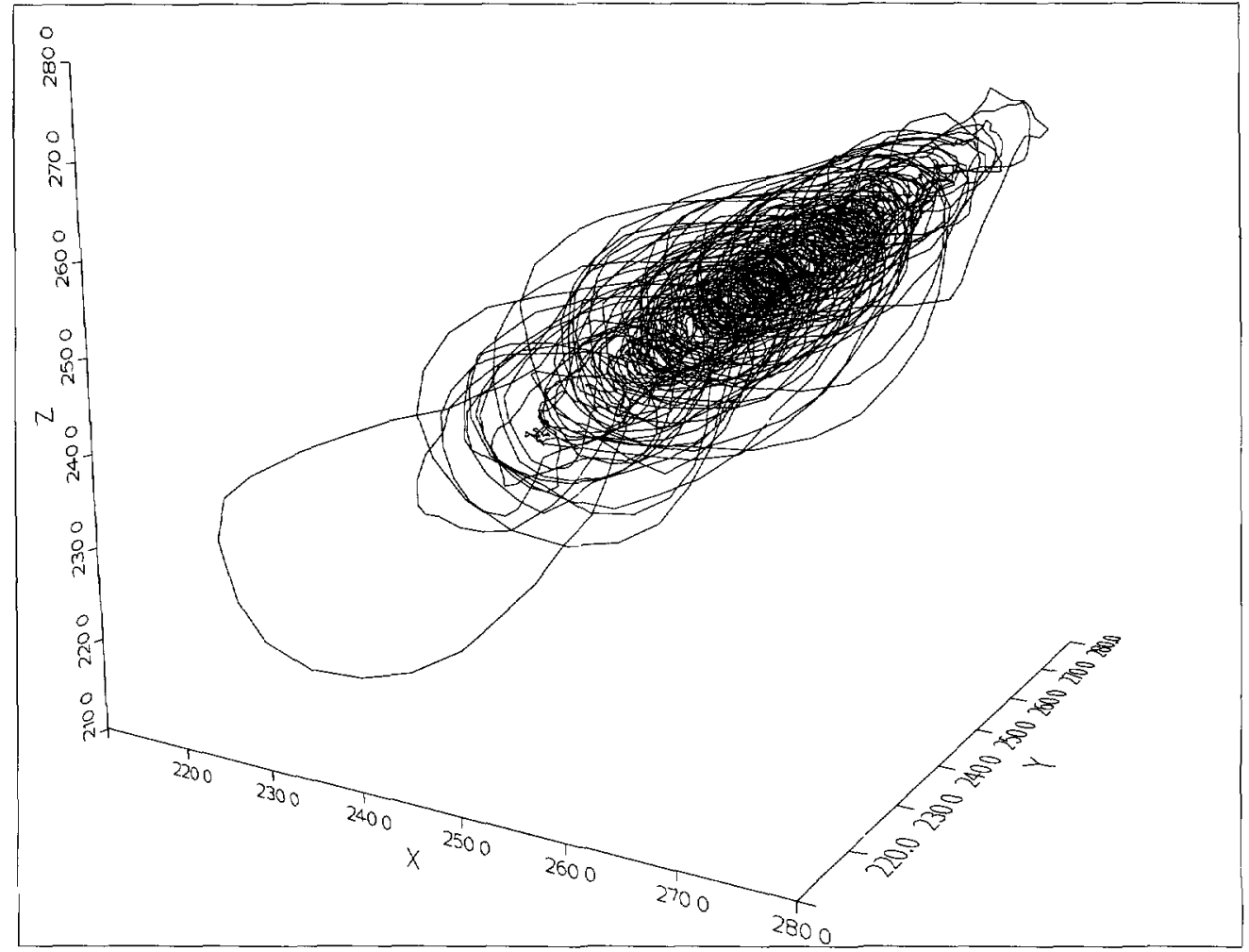

Fig 5. - 3D trajectory reconstructed by the delay method, $p=3, u=0.09 \mathrm{~m} / \mathrm{s}$, for a measurement of absolute pressure at point 2 , at the right-hand side of the bed.

and May [19] to analyse biological tıme series, and also by Wales [20] to extract the largest Lyapunov exponent from a time series. The second one, the surrogate data method, was exposed by Theiler et al. [6]

Both methods only require the knowledge of a scalar time series and are therefore well adapted to the analysis of experimental signals when only one variable is recorded versus time.

3 1. Forecasting Errors. - Given a time series $\left(x_{2}, \imath=1, \ldots n\right)$, the $n_{1}$ first points of the series are used to predict $n_{2}$ other points. The prediction principle is as follows.

A trajectory is constructed by the method of delays (parameters $n, p$ ) Given a reference point, $X_{t}$. we estimate a value $\hat{x}_{t+T}$ of the first coordinate $x_{t+T}$ of the point $X_{t+T}$ at time $t+T$, in which $T$ is the time evolution given as multiples of $\delta t$ Values of $T$ are small when compared to characteristic times of the dynamics This is achieved by selecting the $m$ closest neighbours $X_{1}, \ldots, X_{m}$ of $X_{t}$, and by searching for the linear map $L$ which transforms $X_{2}$ to $x_{\imath+T}$, for $\imath=1, \ldots, m$ The coefficients of the map are found by linear regression (we used a singular value decomposition technique $[27, \mathrm{pp} .52-63])$, and $\hat{x}_{t+T}$ is then taken as the image of $X_{t}$ under $L$.

The forecasting error $E(T)$ is afterwards given by the square root of the averaged sum of the $n_{2}$ values of $\left|\hat{x}_{t+T}-x_{t+T}\right|^{2}$. divided by the standard deviation $\sigma_{x}$ of the $n_{2}$ points $x_{j+T}$, 
so that the forecasting error magnitude does not depend on the signal's scale.

$$
E(T)=\frac{\sqrt{\left.\left(<\left(\hat{x}_{t+T}-x_{t+T}\right)^{2}\right\rangle\right)}}{\sqrt{\left.<(x-<x>)^{2}\right\rangle}}
$$

where the brackets denotes the average on the $n_{2}$ forecasted points.

The behaviour of the forecasting error gives us an indication on the nature of the time series studied a forecasting error which increases exponentially with the forecasting time is a signature of deterministic chaos, whereas a forecasting error which oscillates around a constant is almost certainly the indication that the time series is uncorrelated noise.

Though interesting and easy to implement, this method has severe limitations, because it does not allow any distinction between a stochastic process having strong correlations, also called colored noise, and deterministic chaos. However, by using a very sophisticated prediction technique, let us mention that it is possible [21] to discriminate between colored nolse (powerlaw behaviour), and chaos (exponential behaviour).

3.2. The Method of Surrogate Data - In this method, the studied time series is compared with $N$ surrogate time series, which are stochastic time series constructed to possess some basic features (to be defined) shared with the original one. The principle of the test is that if the studied series and the surrogate ones compare favorably in a sense to be defined later, then it is reasonable to affirm that the studied time series is random. It can then be viewed as a realization of the same random process that had generated the surrogate data. Therefore, if we compute a number representative of each time series (the original and each surrogate ones), the number computed from the original time series must be a realization of the random process generating the numbers computed from the surrogate series.

In practice, the number computed for each time series is called the discriminating statistic of the test. It is noted $Q_{\mathrm{D}}$ for the original time series, while $Q_{\bar{H}}$ will denote the average of the $N$ numbers calculated from the $N$ surrogate ones, with $\sigma_{H}$ the standard deviation. The difference between the studied time series and the surrogate ones is then quantified by a critical ratıo, which is given by:

$$
\mathrm{RC}=\frac{\left|Q_{\mathrm{D}}-Q_{\bar{H}}\right|}{\sigma_{H}}
$$

A very large critical ratio expresses a wide difference between the discriminating statistics of the original time series, and those of the surrogate ones, i.e. a poor comparison between them In this case. when $Q$ is the forecasting error, we reject the hypothesis that the data are stochastic, and decide that they are chaotic. On the contrary, for small values of the critical ratio, the null hypothesis denoted $H_{0}$ has to be accepted, i.e. the original data are stochastic. From the statistical test theory, the threshold value of the critical ratio is taken equal to 2 . The null hypothesis is then accepted for critical ratios smaller than 2 and otherwise rejected With the chosen threshold value, the probability of being wrong, i.e. rejecting $H_{0}$ (data are rejected chaotic) whereas $H_{0}$ is true (data are actually chaotic), is equal to $5 \%$.

The first step of the method is then to construct $N$ random data sets which closely mimic the tested set. There we used a method suggested by Theiler et al. [22], taking for the surrogate data time series having the same Fourier spectrum than the original set, with phases shuffled in the Fourier transform. The resemblance is ensured by the fact that the original and surrogate time series possess the same Fourier spectrum, and thus the same autocorrelation function, but the randomization of the phases of the Fourier transform implies the stochasticity of the surrogate data. We fixed the number of surrogate data sets at $M=40$, ensuring a good enough statistical precision. 
The discriminating statistic of the test might be any number representative of the time series Nevertheless, the use of numbers representing a signature of the non-linearity of the data is obviously highly recommended. Our choice has been the forecasting error which has the advantage of giving a noise/choas distinction test by itself (previous subsection) and which is furthermore not computer time-consuming. Other choice could have been a correlation dimension or a Lyapunov exponent but the significance of these quantities for the surrogate random signals would be doubtful.

Examples of results are now given for some benchmark numerically generated time series i) from Lorentz and Rössler chaotic attractors for the deterministic chaotic case, and ii) from autoregressive processes for the random case. In Figure 6 devoted to the Lorentz system, the forecasting error increases far more slowly for the tested series than for the surrogate series, and consequently the critical ratio is high, equal to or greater than 15 , much larger than the threshold value of 2 Therefore, the null hypothesis $H_{0}$ is rejected and the processed data are chaotic, as expected.

Conversely, Figure 7 displays the case of a 5-degree autoregressive noise. Then the forecasting errors are close for both the original and the surrogate data. The critical ratio is smaller or not significantly bigger than 2 , and we may conclude that the original series is generated by a random process.

3.3. Influence of Parameters - The number of surrogate sets $M$ has been fixed equal to 40 . This choice is a compromise between statistical accuracy requirement and CPU timeconsumption requirements. Nevertheless, since we use the forecasting error method to compute the discriminating statistic, the influence of its parameters has to be investigated. We found that when $n_{1}$ and $n_{2}$ were sufficiently large to ensure statistical precision, a change in the $n_{2}$ value has little influence on the result. But $n_{1}$ must be large enough to be representative of the attractor, and its value thus depends on the sampling rate. For very regular structures, a small number of pseudo-periods is sufficient to ensure realistic precision. We increased the number of points when working on experimental attractors, because of the increasing complexity of the latter.

We will not extensively discuss the role of the parameters $n$ and $p$. it is essentially the same as for the correlation dimension computation, and is thus discussed by several authors [23-26]. As a brief summary, let us recall that the embedding dimension must be large enough to unfold the attractor in the embedding space (Takens criterion). However, the precision of the computation is affected when the embedding dimension is too large, i.e. we have to search for an optimal $n$. Also, the lag time $p$ must be chosen large enough to avoid the trajectory collapsing on the diagonal, but not too large, otherwise decorrelation of the trajectory vector components will occur. The optimal parameters were found through a trial and error process.

Furthermore, there must be enough neighbours $m$ to warrant the accuracy of the computation of the forecasting error. Because of the regression technique used (singular value decomposition), $m$ must be greater than the embedding dimension $n$. On the other hand, we do not possess any theory allowing us to determine an optimal value for $m$. It can just be sald that the greater the number of neighbours, the greater the accuracy of forecasting, but that taking too many neighbours will lead us to consider as neighbours points of the trajectory which are in reality quite far from the reference point and will spoil the computation of the forecasting error, 1.e here again we have to search for an optimal value. This effect can be seen in Figure 8 where we have indicated the forecasting error, for the Lorentz system, for different values of $m$, the other parameters being kept constant An optimum (i.e. a minimum) of the forecasting error is obtained for $m=50$. Nevertheless, though the value of $m$ influences the forecasting error, the repercussion of this influence on the result of the test is negligible. In 

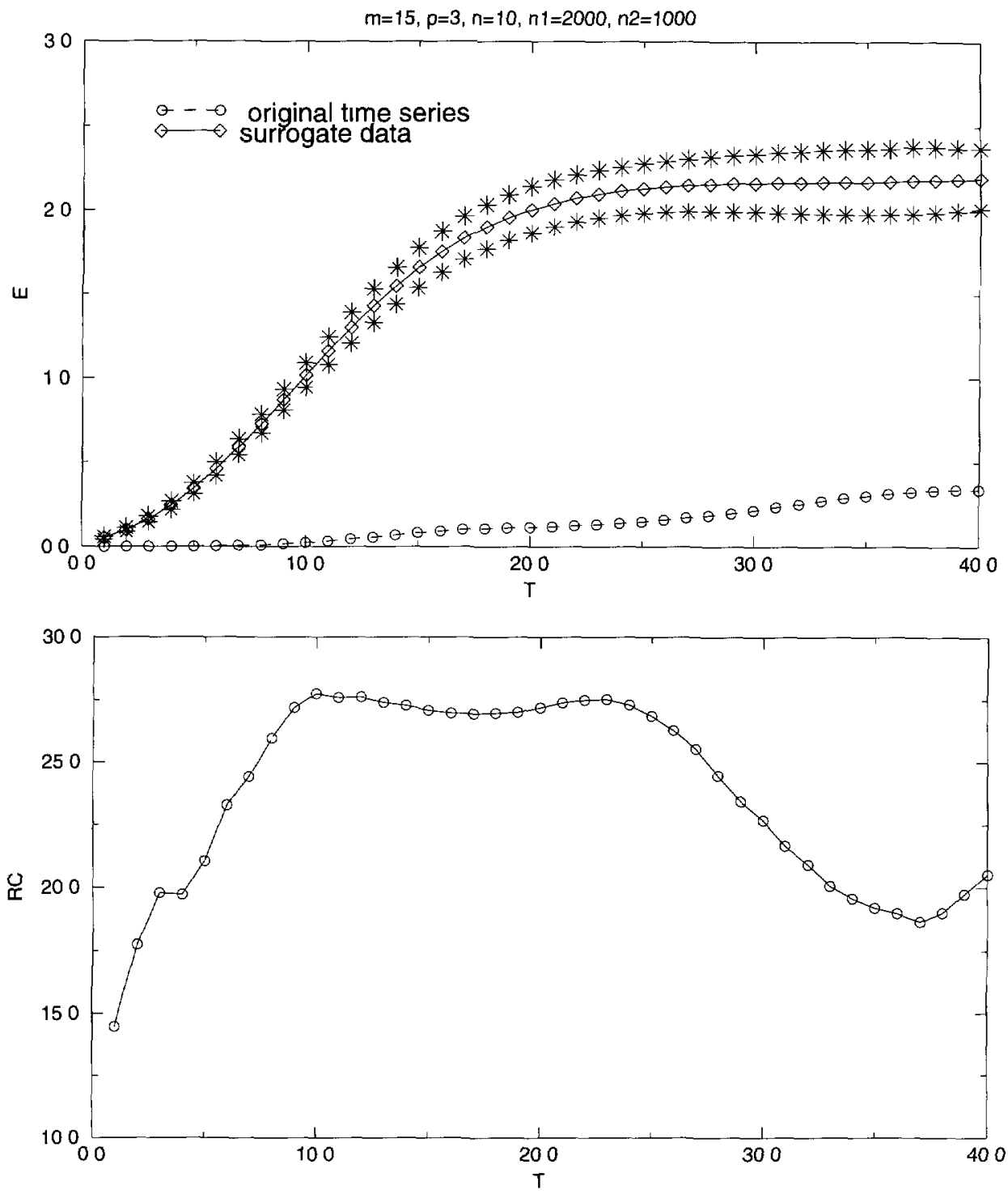

Fig. 6. - Forecasting error, and critical ratio, for the Lorentz process, errors bars are represented by the symbol $(\star) T$ is the time evolution given as multıples of $\delta t$.

fact, the effects are quite similar on the forecasting errors of both the studied signal and of the surrogate data, so that the comparison is not significantly modified by the parameter $m$. For instance, we have tested several values of $m$ for the Lorentz process Whatever $m$, the null hypothesis $H_{0}$ is rejected, i.e. the Lorentz process was found to be chaotic. Conversely, the same test applied to an autoregressive noise always led us to the acceptance of the null hypothesis $H_{0}$, regardless of the value of $m$, i.e. the process was found to be random.

We also investigated the effect of noise in the case of the Lorentz system, by modifying time series with additive noises of various amplitudes $(2,5$, and $50 \%$ of the amplitude of the 

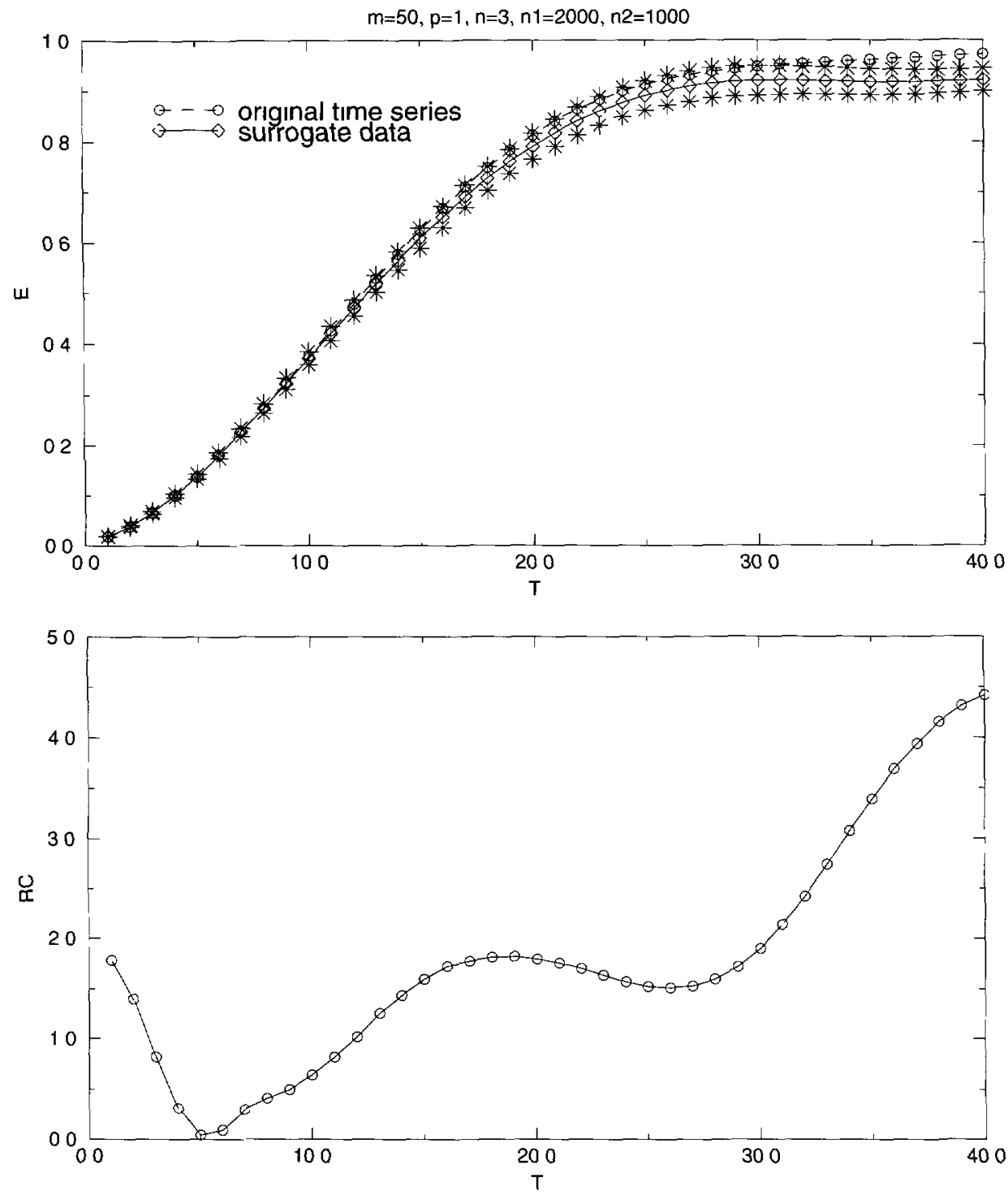

Fig 7. - Forecasting error, and critical ratio, for a 5-degree autoregressive noise.

process). We found that the surrogate data method did not work in the third case, but was still valid for the first two cases. which correspond to many expermental situations. Thus, the method of surrogate data can be considered as reliable and robust with respect to noise and changes in the parameters values.

\section{Application to the Experimental Series}

A typical example of the results is displayed in Figure 9 The difference of forecasting error between experimental and surrogate data is small. leading us to the conclusion that the data is 


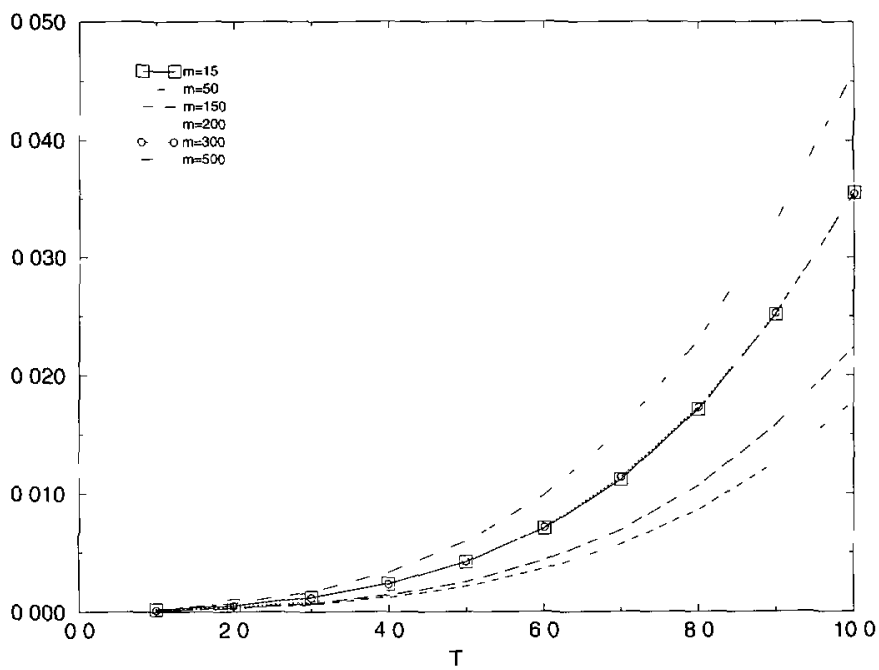

Fig. 8 - Forecasting error computed for the Lorentz process. for different numbers of neighbours $m$. $n=10, p=3 ; n_{1}=2000, n_{2}=500$
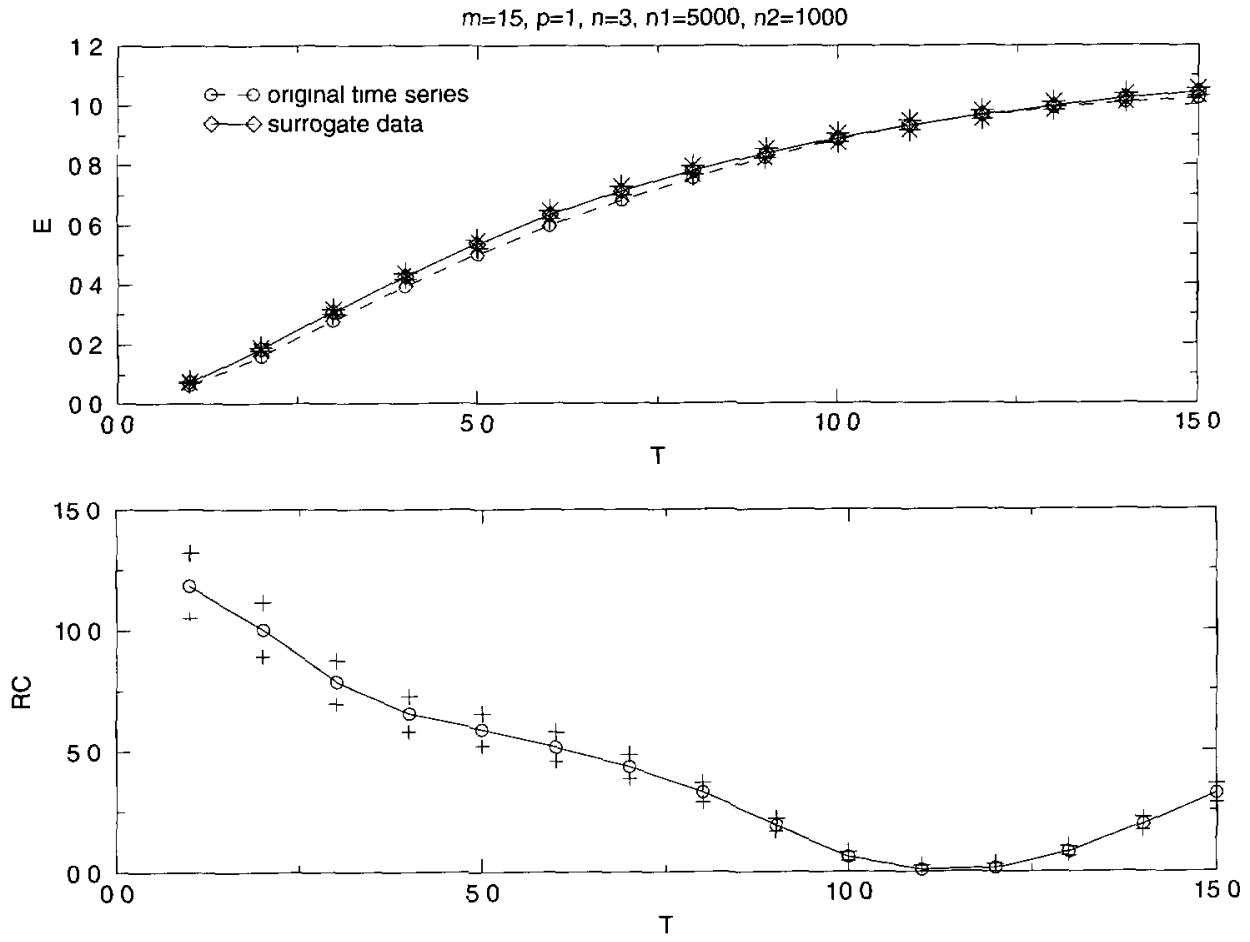

Fig. 9. - Forecastıng error. and critıcal ratio, for a recordıng of absolute pressure, on the left, at the tap number two, with a superficial velocity $u=0.12 \mathrm{~m} / \mathrm{s}$ 

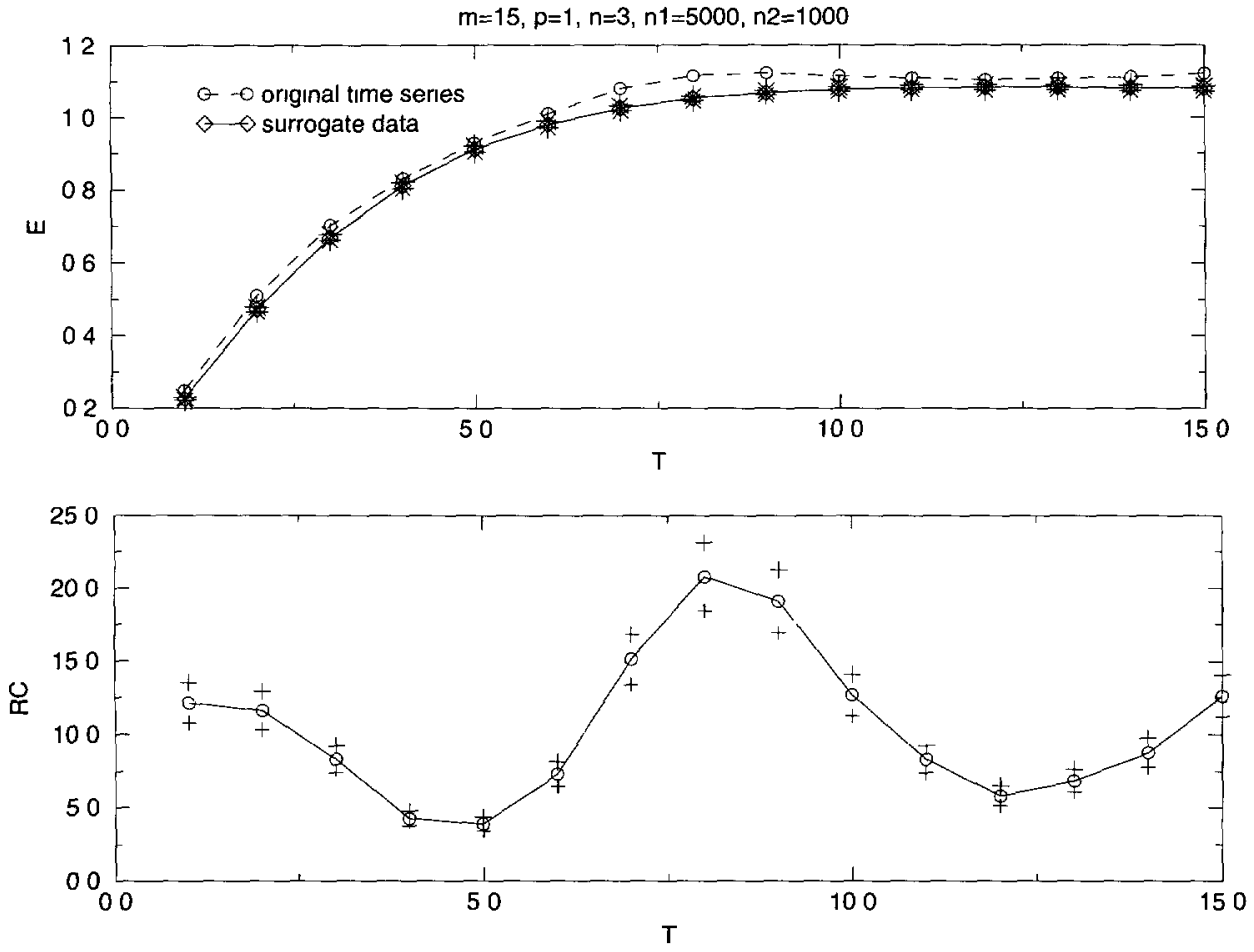

Fig. 10. - Forecasting error, and critical ratio, for the same recording, sampled at every second point.

not produced by a low-dimensional chaotic system. But, on the other hand, the critical ratio corresponding to this case is large, due to a very small dispersion around the average for the surrogate data error (denominator in relation (2)). Strictly speaking, we ought to reject the null hypothesis and claim for chaos, but the conflict between both conclusions must lead us to refine our analysis.

Let us remove every second point from the initial tıme series. In this way, the sampling rate is now divided by two. We apply the method of surrogate data on this new time series, without modifying any parameter. Then, the whole procedure is repeated, so, at the next step, the time series is numerically sampled at a rate which is four times smaller than the initial one.

The results we obtained for the time series sampled at every second point show a forecasting error for the experimental data which is larger (or let us say very similar to) than for the surrogate data (Fig. 10), indicating stochasticity This conclusion is still conflicting with the conclusion we would draw from the critical ratio.

However, for the time series sampled at every fourth point. Figure 11 shows that the forecasting error for the experimental and the surrogate data are similar and that the critical ratio is about two or smaller than two at small forecasting times. This last result. taken together with the preceding results, clearly indicates the stochasticity of the data.

Note that our procedure of removing points in the time series is equivalent to a decrease of the acquisition frequency and somehow in the same spirit than the Theiler procedure [22] of removing neighbours in space which are also neighbours in time. Also, the influence of the sampling time here may be understood as follows the size of the experimental files is fixed, being limited by the processing time. Therefore, a small sampling time leads us to analyze 

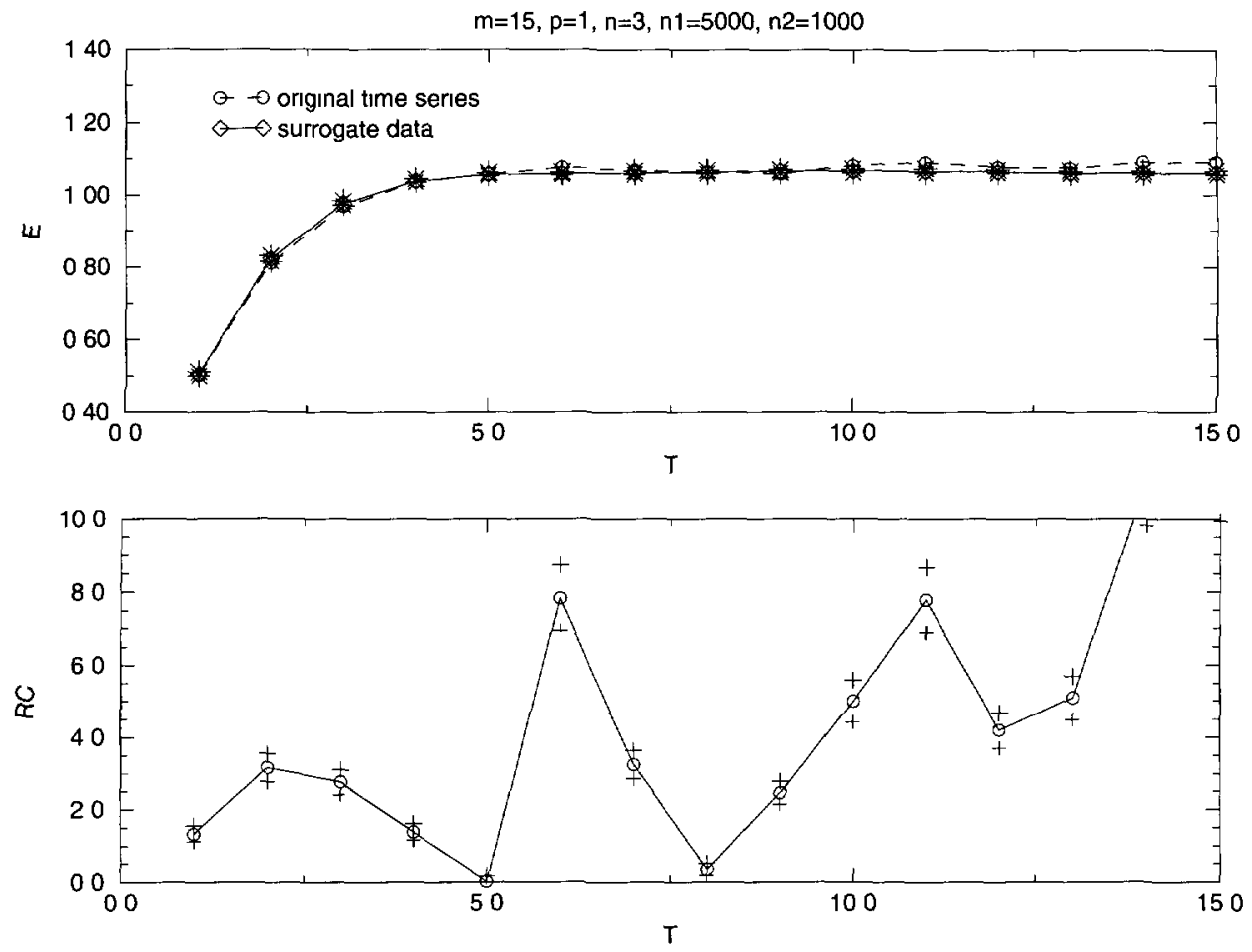

Fig. 11. - Forecastıng error, and critıcal ratio, for the same recording, sampled at every fourth point.

a small portion of trajectory. Increasing the sampling time conversely allows us to analyze a larger portion of the trajectory, i.e. to obtam more relevant information on the object under study.

4.1 The Effects of Smoothing. - Many experimenters use smoothing techniques before processing their data. Let us examine the effects of one of these techniques [27, pp. 495-497], which removes any linear trend, and then uses a Fast Fourier Transform to low-pass the data. The linear trend is reinserted at the end of the process. The amount of smoothing is defined by a constant $K$. Then, the cut-off frequency is given by $f_{\mathrm{c}} \approx n_{\mathrm{q}} / K \times f_{\mathrm{s}}$ where $f_{\mathrm{s}}$ is the sampling rate, and $n_{\mathrm{q}}$ the greater power of two which is smaller than or equal to the size of the data record.

As shown in Figure 12, the results obtained for a smoothed experimental time series clearly indicate a strong difference for the forecasting error between experimental and surrogate data, and the critical ratio is large. Two conflicting interpretations may then a proro be proposed:

- The signal is deterministic and chaotic. But the non-linear features were masked by the experimental noise.

- The signal is stochastic, but the smoothing procedure introduced low-frequency effects which falsify the results.

We tested these assumptions by applying the same procedure to the analysis of an autoregressive noise. For the untreated process, we obtained a critical ratio smaller than two, and a forecasting error very close to surrogate forecasting error, indicating stochasticity as it should. 

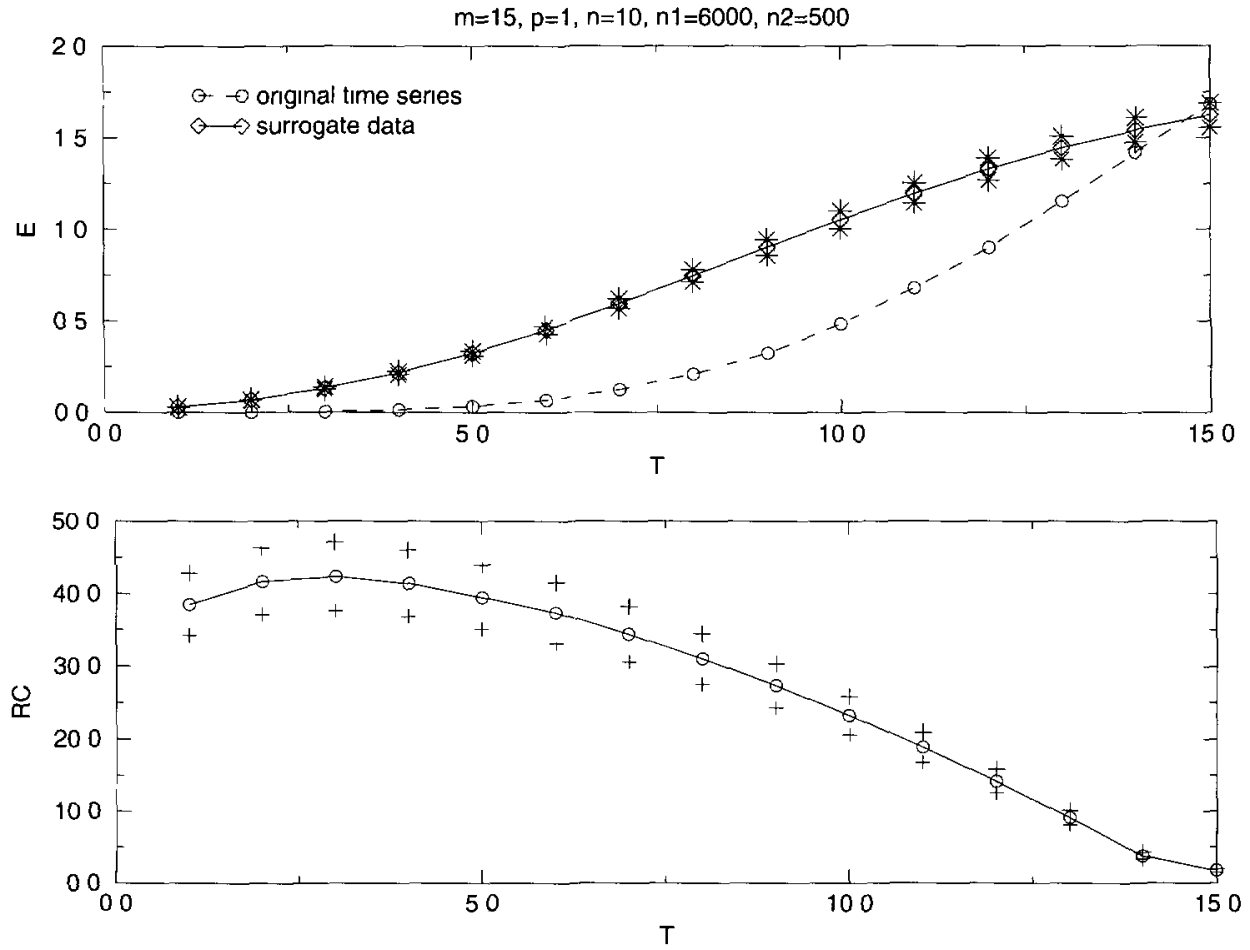

Fig. 12 - Forecastıng error, and critical ratı, for a smoothed recording of pressure.

On the contrary, when the time series was smoothed, results displayed in Figure 13 would have lead us to reject the null hypothesis and to conclude to the presence of chaos. which would be a spurious conclusion. Therefore, the use of a noise reduction technique may falsify the results given by the surrogate data method Our final conclusion on the distinction between noise and chaos for our data is then that the experimental time series measured in the fluidized bed are not generated by a low-dimensional process, but can be the results of a stochastic process. The next step is then to ask whether we can characterize this process.

\section{Hurst Analysis}

Some stochastic processes exhibit long-term statistical dependence, among them the fractal Brownian motions ( $\mathrm{fBm})$, which verify [28]:

- the increments $B_{H}\left(t_{2}\right)-B_{H}\left(t_{1}\right)$ and $B_{H}\left(t_{4}\right)-B_{H}\left(t_{3}\right)$ are independent

- an increment $B_{H}\left(t_{2}\right)-B_{H}\left(t_{1}\right)$ follows a Gaussian law, with zero average and a standard deviation proportional to $\left|t_{2}-t_{1}\right|$.

The main property of the $\mathrm{fBm}$ is related to the value of the correlation $C(t)$ between $B_{H}(-t)$ and $B_{H}(t)$, given by:

$$
C(t)=\left(2^{2 H-1}-1\right)
$$

Thus, we can distinguish three cases: 

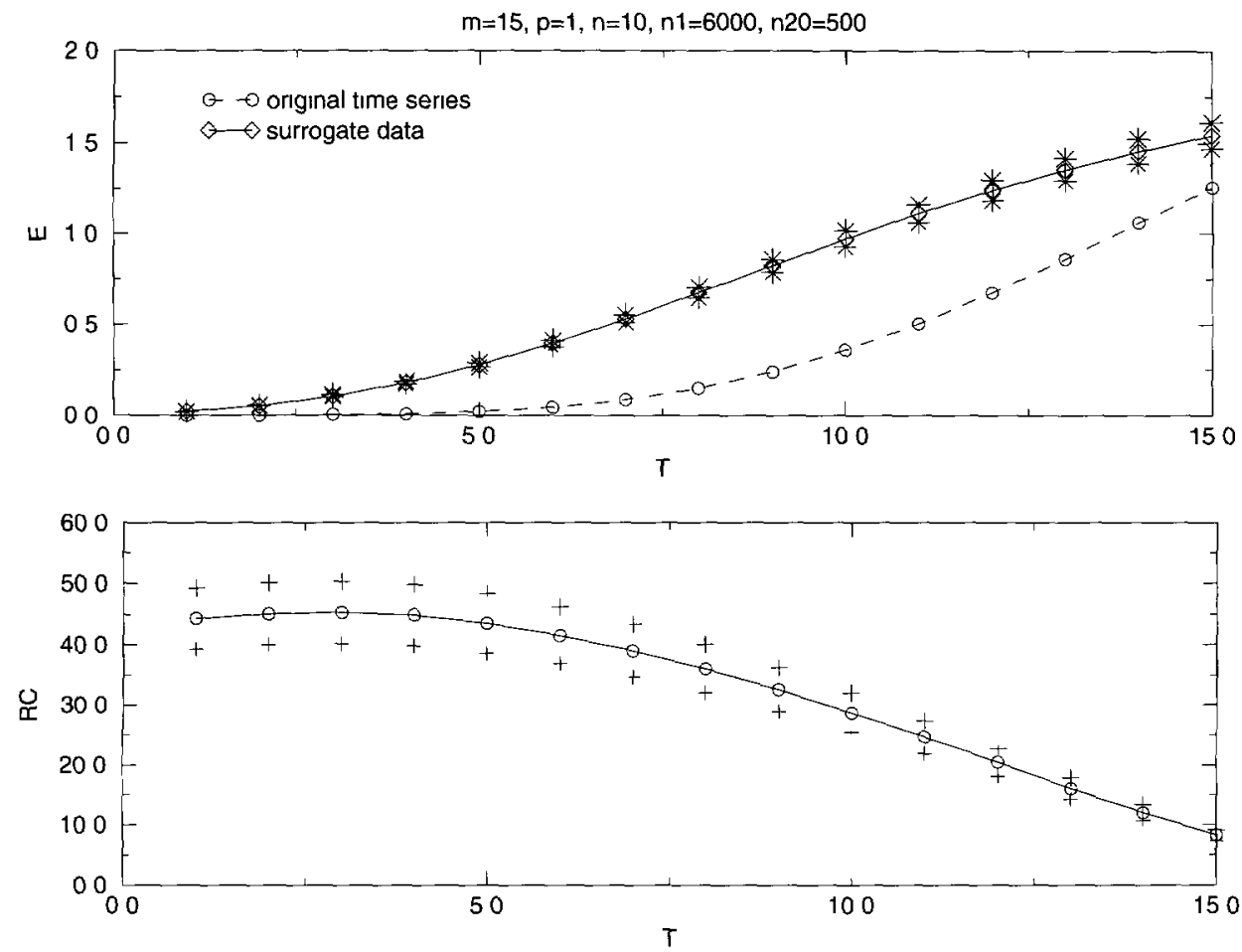

Fig. 13. - Forecasting error, and critical ratio, for a smoothed autoregressive noise.

- $H=0.5$ The correlation between $B_{H}(-t)$ and $B_{H}(t)$ is always zero, i.e. the process is a Brownian motion, as defined by Wiener in 1923 [29], with no memory.

- $H>0.5$. The correlation is always positive, i e. the trends of the graph at the time $-t$ are conserved at the time $t$. Large excursions appear on the curves giving $B_{H}(t)$ versus $t$, and the signal is said to be persistent.

- $H<0.5$. Here exactly the contrary is true The signal is said to be antipersistent, and the curves appear to be much more crinkly.

Hurst's analysis [30,31] allows the $H$-value to be deduced from a time series, as follows. First, from the time series $\left(x_{2}\right)$, considered on a total lag time $\tau$, the cumulative departure $X(t, \tau)$ to the average is computed:

$$
X(t, \tau)=\sum_{\imath=1}^{t} x_{\imath}-\langle x\rangle_{\tau}
$$

Then we define a range $R$ which is equal to the maximum of the cumulative departure minus its minimum, and depends only on $\tau$ :

$$
R(\tau)=\max _{1 \leq t \leq \tau} X(t)-\min _{1 \leq t \leq \tau} X(t)
$$

Finally, the quantity $R / S$. where $S$ is the mean square devation of the time series, follows the empirical law.

$$
R / S \propto(\alpha \tau)^{H}
$$




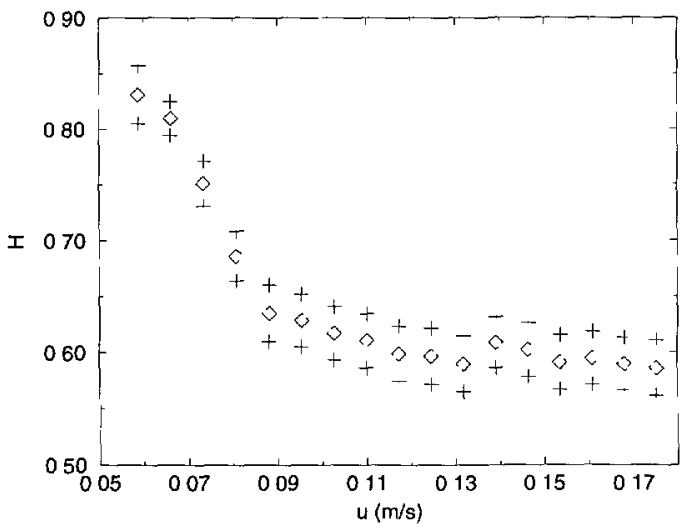

Fig. 14. - Hurst's exponent versus superficial velocity $(\diamond)$, for measurement of right-hand absolute pressure, at point 4 , the symbol $(+)$ indicate error measurement on $H$.

Therefore, by plotting $\log (R / S)$ for different lag times $\tau$, the value of $H$ can be evaluated by linear regression.

5.1. Application to the Experiments. - We calculated the value of $H$, and plotted it versus superficial velocity, as shown in Figure 14. For a small velocity, $H$ is quite large $(\approx 0.8)$, i e. strong correlations are present in the signal, corresponding to very low frequency effects (the signal is persistent). $H$ decreases with an increase in the superficial velocity, and eventually reaches values around 0.6 , which is not far from the value expected for a Brownian motion If we consider the fractal dimension associated to $H\left(D_{H}=1 / H\right)$, the latter increases with velocity, representing the fact that the number of bubbles in the bed increases, and that the re-circulation time of the particles decreases.

We noticed that the values found for $H$, for different taps, and for differential pressures as well as for absolute pressures are quite close, so that the difference between them can be due to measurement uncertainties In this case, it would be possible to associate to a given $H$ a bubbling intensity, and therefore to find a way of controlling the bubbling.

\section{Conclusion}

To analyse pressure measurement in a fluidized bed, we used several techniques to discriminate between noise and chaos. In particular, from results obtained by using a surrogate data method, we concluded that the experimental time series generated by the fluidized bed were probably random, and at least were not low-dimensional chaos. It has been illustrated that much care must be taken to avoid spurious identification of deterministic chaos.

In particular, a nalve use of correlation dimension evaluations may be misleading due to the fact that some random processes, characterized by strong correlations, may lead to misleading low-dimension effects. Once our process has been assumed to being random, Hurst's analysis allowed us to suggest that the random process could possibly be, as a first approximation, a fractal Brownian motion characterized by a Hurst exponent $H$. The complicated dynamics exhibited by the fluidization process may then be characterized by a single number. The use of such a synthetic number could allow the identification of fluidization regimes.

It should however be noted that our study has been carried out in terms of a noise/chaos 
distinction. In many cases, both aspects are mixed. Therefore, if there exists a deterministic component in our signals, a more refined conclusion would be that this component could not be detected by our analysis, i.e the dynamics of the fluidized bed is dominated by randomness.

\section{References}

[1] Van Den Bleek C M. and Schouten J C., Chem. Eng J 53 (1993).

[2] Tam S.W and Devine M.K, Is there a strange attractor in a flundized bed ?, in Measures of Complexity and Chaos, N.B Abraham et al Eds., (Plenum Press, New York)

[3] Provenzale A, Smith L.A., Vio R. and Murante G, Physıca D 58 (1992) 31-49.

[4] Meunier-Guttın-Cluzel S, Maheu B, Gouesbet G, Physıca D 58 (1992).

[5] Rapp P E., Albano A M , Schmah T.I., Farwell L.A., Phys Rev E 47 (April 1993)

[6] Theiler J., Eubank S., Longtin A, Galdrikıan B and Farmer J.D., Using surrogate data to detect nonlinearity in time series, , in Nonlınear prediction and modeling, M. Casdaglı and S. Eubanks Eds., (Addison-Wesley, 1992).

[7] Theiler J , Phys Rev A 34 (1986) 2427-2432.

[8] Throye M., Darrigo R and Gouesbet G, Monte-Carlo sımulation of bubbling fluidized beds, in Instabilities in Multiphase Flows, G. Gouesbet and A. Berlemont Eds, (Plenum Press, 1993)

[9] Letaief $N$, Distınction brut/chaos applıquée à l'étude d'un lit fluidisé bouillonnant, Thèse de Doctorat de l'Unıversité de Rouen, France (Janvier 1990).

[10] Fan Z, Chen G T, Chen B.C. and Yuan H., Powder Tech. 62 (1990) 139-145.

[11] Neogi D., Fan L T, Yutani N., Nassar R and Walawender W P., Apl. Stoch. Models Data Analysis 4 (1988) 13-34.

[12] Kage H , Iwasaki N., Yamaguchi H and Matsuno Y., J. Chem. Eng Jpn 24 (1) (1991).

[13] Saxena S.C. and Rao N S., Energy 15 (1990) 489-497.

[14] Huang Y.W., Fan L.T, Song J.C. and Yutani N, Pressure fluctuation in a gas-solıd fludized bed in a screen

[15] Drahos J., Bradka F and Puncochar M, Chem Eng. Scı 47 (1992) 4069-4075.

[16] Grassberger P and Procaccia I, Physıca D 9 (1983) 189.

[17] Gouesbet G.. Phys Rev. A 42 (1990) 5928-5945.

[18] Farmer J D and Sidorowich J.J, Phys Rev. Lett. 59 (August 1987).

[19] Sugihara G and May R.M., Nature 334 (Avrıl 1990).

[20] Wales D.J., Nature 350 (Aprll 1991).

[21] Tsonis A.A and Elsner J.B, Nature 358 (July 1992).

[22] Theiler J., Eubank S, Longtin A, Galdrikıan B and Farmer J.D., Physica D 58 (1992).

[23] Buzug Th. and Pfister G., Phys. Rev. A 45 (Mal 1992).

[24] Aleksie Z, Physıca D 52 (1991).

[25] Liebert W and Schuster H G., Phys. Lett. A 142 (December 1989).

[26] Buzug Th. and Pfister G., Europhys Lett 13 (December 1990)

[2T] Press W.H, Flannery B P., Teukolsky S.A and Vetterling W.T., Numerical Recipes (Cambridge University Press, 1986).

[28] Mandelbrot B.B. and van Ness J.W., SIAM Rev. 10 (October 1968)

[29] Wiener N., J Math Phys. Mass. Inst. Technol. 2 (1923)

[30] Hurst H E., Trans. Am Soc. Cvv Eng 116 (1951).

[31] Feder J, Fractals (Plenum Pub. Corp., New York, 1988). 